S17 Table. Cartesian coordinates of analyzed rotamers (adducts)

$\begin{array}{lrrr}\text { Rotamer I } & & \\ \text { O 1 } & & & \\ \mathrm{C} & 3.386127000 & -2.002026000 & 0.206338000 \\ \mathrm{C} & 2.220036000 & -2.265955000 & 0.905428000 \\ \mathrm{~N} & 1.164983000 & -1.447234000 & 0.979609000 \\ \mathrm{C} & 1.243913000 & -0.265234000 & 0.332858000 \\ \mathrm{C} & 2.393148000 & 0.112240000 & -0.395452000 \\ \mathrm{C} & 3.475555000 & -0.769238000 & -0.470977000 \\ \mathrm{~N} & 0.138468000 & 0.562907000 & 0.424020000 \\ \mathrm{C} & -1.008830000 & 0.202095000 & 1.262939000 \\ \mathrm{C} & 0.034133000 & 1.797853000 & -0.334012000 \\ \mathrm{~N} & 4.593671000 & -0.457917000 & -1.226598000 \\ \mathrm{C} & -2.210800000 & -0.297970000 & 0.479908000 \\ \mathrm{C} & -2.132671000 & -1.491505000 & -0.253797000 \\ \mathrm{C} & -3.234491000 & -1.954462000 & -0.969785000 \\ \mathrm{C} & -4.432526000 & -1.233568000 & -0.965462000 \\ \mathrm{C} & -4.521012000 & -0.047760000 & -0.238329000 \\ \mathrm{C} & -3.414250000 & 0.415067000 & 0.478523000 \\ \mathrm{C} & 0.632981000 & 3.003911000 & 0.395834000 \\ \mathrm{O} & 0.454073000 & 4.132275000 & -0.451040000 \\ \mathrm{H} & 4.198177000 & -2.721303000 & 0.177755000 \\ \mathrm{H} & 2.118974000 & -3.207230000 & 1.444444000 \\ \mathrm{H} & 2.458205000 & 1.072214000 & -0.893377000 \\ \mathrm{H} & -0.666056000 & -0.566543000 & 1.956137000 \\ \mathrm{H} & -1.300736000 & 1.082688000 & 1.849091000 \\ \mathrm{H} & 0.512751000 & 1.692772000 & -1.312487000 \\ \mathrm{H} & -1.023665000 & 1.996301000 & -0.532099000 \\ \mathrm{H} & 4.736727000 & 0.519040000 & -1.437903000 \\ \mathrm{H} & 5.445248000 & -0.942682000 & -0.984004000 \\ \mathrm{H} & -1.203170000 & -2.052934000 & -0.247366000 \\ \mathrm{H} & -3.161906000 & -2.882179000 & -1.530705000 \\ \mathrm{H} & -5.290003000 & -1.596554000 & -1.524911000 \\ \mathrm{H} & -5.447436000 & 0.519665000 & -0.229009000 \\ \mathrm{H} & -3.488734000 & 1.339228000 & 1.047604000 \\ \mathrm{H} & 0.122967000 & 3.134833000 & 1.363223000 \\ \mathrm{H} & 1.696449000 & 2.818529000 & 0.610642000 \\ \mathrm{H} & 0.791899000 & 4.910975000 & 0.007972000\end{array}$

\title{
Rotamer Ib
}

11

C $\quad 16.624110000 \quad-25.340922000 \quad-1.562465000$

C $\quad 16.711435000 \quad-26.692465000 \quad-1.652756000$

$\begin{array}{llll}\mathrm{N} & 17.871688000 & -27.353362000 & -1.365564000\end{array}$

C $\quad 19.012910000 \quad-26.703421000 \quad-0.956062000$

C $\quad 18.958738000 \quad-25.306119000 \quad-0.850724000$

C $\quad 17.778491000 \quad-24.605712000 \quad-1.141337000$

N $\quad 20.107133000 \quad-27.459292000 \quad-0.683562000$ 


$\begin{array}{lrrr}\mathrm{C} & 20.171115000 & -28.880421000 & -1.083015000 \\ \mathrm{C} & 21.332708000 & -26.839337000 & -0.164625000 \\ \mathrm{~N} & 17.717725000 & -23.263261000 & -1.045356000 \\ \mathrm{C} & 19.228139000 & -29.809695000 & -0.330713000 \\ \mathrm{C} & 19.072266000 & -29.720234000 & 1.060011000 \\ \mathrm{C} & 18.238389000 & -30.611699000 & 1.732007000 \\ \mathrm{C} & 17.550026000 & -31.601931000 & 1.024897000 \\ \mathrm{C} & 17.696957000 & -31.696841000 & -0.358218000 \\ \mathrm{C} & 18.531216000 & -30.802252000 & -1.034437000 \\ \mathrm{C} & 22.274738000 & -26.317385000 & -1.255987000 \\ \mathrm{O} & 23.304461000 & -25.640583000 & -0.565755000 \\ \mathrm{H} & 15.695762000 & -24.837513000 & -1.802447000 \\ \mathrm{H} & 15.879431000 & -27.318553000 & -1.950254000 \\ \mathrm{H} & 19.844619000 & -24.756631000 & -0.566087000 \\ \mathrm{H} & 19.992062000 & -28.966146000 & -2.162706000 \\ \mathrm{H} & 21.206378000 & -29.190609000 & -0.920325000 \\ \mathrm{H} & 21.075030000 & -26.020943000 & 0.511479000 \\ \mathrm{H} & 21.857205000 & -27.582508000 & 0.440046000 \\ \mathrm{H} & 18.510328000 & -22.720771000 & -0.739690000 \\ \mathrm{H} & 16.870996000 & -22.761818000 & -1.261835000 \\ \mathrm{H} & 19.603331000 & -28.953614000 & 1.617218000 \\ \mathrm{H} & 18.127579000 & -30.537465000 & 2.809232000 \\ \mathrm{H} & 16.903414000 & -32.295790000 & 1.552384000 \\ \mathrm{H} & 17.168209000 & -32.465329000 & -0.913052000 \\ \mathrm{H} & 18.655752000 & -30.891045000 & -2.111640000 \\ \mathrm{H} & 22.653339000 & -27.160466000 & -1.853725000 \\ \mathrm{H} & 21.724156000 & -25.653186000 & -1.941603000 \\ \mathrm{H} & 24.019699000 & -25.435082000 & -1.180978000 \\ \mathrm{H} & 17.851086000 & -28.368399000 & -1.320756000\end{array}$

\section{Rotamer Ic}

01

C $\quad 16.781036000 \quad-24.646955000 \quad-1.599579000$

C $\quad 18.071810000 \quad-24.339632000 \quad-1.211826000$

N $\quad 19.042295000 \quad-25.241240000 \quad-1.001642000$

C $\quad 18.732342000 \quad-26.546831000 \quad-1.157427000$

C $\quad 17.438049000 \quad-26.966798000 \quad-1.524507000$

$\begin{array}{llll}\text { C } & 16.448313000 & -26.008855000 & -1.764113000\end{array}$

$\begin{array}{llll}\mathrm{N} & 19.749975000 & -27.452867000 & -0.928359000\end{array}$

C $\quad 19.676857000 \quad-28.811912000 \quad-1.434130000$

C $\quad 20.963948000 \quad-27.075063000 \quad-0.189789000$

N $\quad 15.183772000 \quad-26.380461000 \quad-2.176462000$

C $\quad 19.143241000 \quad-29.866293000 \quad-0.466961000$

$\begin{array}{llll}\text { C } & 18.579880000 & -29.528508000 & 0.766443000\end{array}$

$\begin{array}{llll}\text { C } & 18.093863000 & -30.521316000 & 1.621489000\end{array}$

C $\quad 18.164391000 \quad-31.863711000 \quad 1.252320000$

$\begin{array}{llll}\text { C } & 18.726708000 & -32.210498000 & 0.020895000\end{array}$

$\begin{array}{llll}\text { C } & 19.213109000 & -31.219012000 & -0.828601000\end{array}$

$\begin{array}{llll}\text { C } & 22.064871000 & -26.396014000 & -1.018734000\end{array}$

O $\quad 21.842658000 \quad-25.024988000 \quad-1.244565000$ 


$\begin{array}{lrrr}\mathrm{H} & 16.049975000 & -23.864483000 & -1.773892000 \\ \mathrm{H} & 18.362171000 & -23.300440000 & -1.070880000 \\ \mathrm{H} & 17.189457000 & -28.020283000 & -1.578309000 \\ \mathrm{H} & 19.071387000 & -28.814194000 & -2.347890000 \\ \mathrm{H} & 20.684704000 & -29.106701000 & -1.752952000 \\ \mathrm{H} & 20.688309000 & -26.408142000 & 0.633209000 \\ \mathrm{H} & 21.357051000 & -27.996765000 & 0.252867000 \\ \mathrm{H} & 14.913464000 & -27.339341000 & -2.013699000 \\ \mathrm{H} & 14.440706000 & -25.716433000 & -2.017423000 \\ \mathrm{H} & 18.523595000 & -28.484037000 & 1.055882000 \\ \mathrm{H} & 17.661194000 & -30.241764000 & 2.577977000 \\ \mathrm{H} & 17.788135000 & -32.635308000 & 1.917532000 \\ \mathrm{H} & 18.790019000 & -33.253955000 & -0.274597000 \\ \mathrm{H} & 19.654862000 & -31.497161000 & -1.783371000 \\ \mathrm{H} & 23.012327000 & -26.495635000 & -0.469684000 \\ \mathrm{H} & 22.187312000 & -26.956417000 & -1.964625000 \\ \mathrm{H} & 20.869905000 & -24.896830000 & -1.232710000\end{array}$

\section{Rotamer Id}

01

C $\quad 16.540644000 \quad-24.789582000 \quad-1.641047000$

$\begin{array}{llll}\text { C } & 16.338799000 & -26.141629000 & -1.863378000\end{array}$

$\begin{array}{llll}\mathrm{N} & 17.290282000 & -27.080404000 & -1.811584000\end{array}$

C $\quad \begin{array}{llll}18.548008000 & -26.681503000 & -1.529898000\end{array}$

$\begin{array}{llll}\text { C } & 18.866424000 & -25.326686000 & -1.296666000\end{array}$

C $\quad 17.851265000 \quad-24.365670000 \quad-1.344280000$

$\begin{array}{llll}\mathrm{N} & 19.520476000 & -27.671209000 & -1.503340000\end{array}$

C $\quad 19.154463000 \quad-29.086906000 \quad-1.682301000$

C $\quad 20.874209000 \quad-27.403811000 \quad-1.025851000$

N $\quad 18.118157000 \quad-23.036788000 \quad-1.063569000$

C $\quad 18.993942000 \quad-29.860551000 \quad-0.384489000$

$\begin{array}{llll}\text { C } & 17.949836000 & -29.562867000 & 0.504704000\end{array}$

$\begin{array}{llll}\text { C } & 17.809512000 & -30.274709000 & 1.694396000\end{array}$

C $\quad 18.708113000 \quad-31.296122000 \quad 2.016211000$

$\begin{array}{llll}\text { C } & 19.746574000 & -31.602481000 & 1.138245000\end{array}$

C $\quad 19.886388000 \quad-30.886705000 \quad-0.053621000$

C $\quad 21.811914000 \quad-26.917465000 \quad-2.139533000$

O $\quad 21.841765000 \quad-27.814682000 \quad-3.239920000$

$\mathrm{H} \quad 15.717209000 \quad-24.084425000-1.688640000$

$\mathrm{H} \quad 15.338481000 \quad-26.503505000 \quad-2.097736000$

$\mathrm{H} \quad 19.880913000 \quad-25.011071000-1.086552000$

$\mathrm{H} \quad 18.223646000 \quad-29.103807000 \quad-2.249527000$

$\mathrm{H} \quad 19.939776000 \quad-29.554713000 \quad-2.284553000$

$\begin{array}{llll}\mathrm{H} & 20.860499000 & -26.683593000 & -0.198077000\end{array}$

$\mathrm{H} \quad 21.267910000 \quad-28.338741000 \quad-0.617698000$

$\mathrm{H} \quad 19.078603000 \quad-22.739365000 \quad-1.155818000$

$\mathrm{H} \quad 17.455420000 \quad-22.363002000 \quad-1.418179000$

H $\quad 17.247073000 \quad-28.776451000 \quad 0.247348000$

$\mathrm{H} \quad 16.994049000 \quad-30.036525000 \quad 2.371788000$

H $\quad 18.595705000 \quad-31.849874000 \quad 2.943966000$ 


$\begin{array}{llll}\mathrm{H} & 20.448723000 & -32.396055000 & 1.378090000 \\ \mathrm{H} & 20.694697000 & -31.132869000 & -0.738646000 \\ \mathrm{H} & 21.526744000 & -25.906244000 & -2.467170000 \\ \mathrm{H} & 22.835472000 & -26.861970000 & -1.753427000 \\ \mathrm{H} & 20.947391000 & -27.818349000 & -3.608719000\end{array}$

\section{Rotamer Ie}

01

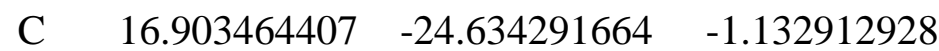

$\begin{array}{llll}\text { C } & 16.630116260 & -25.901136675 & -1.643602498\end{array}$

$\begin{array}{llll}\mathrm{N} & 17.526585257 & -26.898800227 & -1.701359804\end{array}$

$\begin{array}{llll}\text { C } & 18.799602607 & -26.656956788 & -1.248374973\end{array}$

$\begin{array}{llll}\text { C } & 19.173265959 & -25.410764128 & -0.709650716\end{array}$

$\begin{array}{llll}\text { C } & 18.210781210 & -24.389848334 & -0.658332368\end{array}$

$\begin{array}{llll}\mathrm{N} & 19.725378537 & -27.676361991 & -1.442269173\end{array}$

C $\quad \begin{array}{llll}19.259091075 & -29.016891067 & -1.837110405\end{array}$

$\begin{array}{llll}\text { C } & 20.964964307 & -27.658989676 & -0.649953781\end{array}$

$\begin{array}{llll}\mathrm{N} & 18.555552580 & -23.150039386 & -0.165689997\end{array}$

C $\quad 18.943740136 \quad-29.899260596 \quad-0.656170808$

$\begin{array}{llll}\text { C } & 17.797422907 & -29.661027494 & 0.103283887\end{array}$

$\begin{array}{llll}\text { C } & 17.514334131 & -30.461176713 & 1.206803400\end{array}$

$\begin{array}{llll}\text { C } & 18.370770647 & -31.506942912 & 1.549222716\end{array}$

$\begin{array}{llll}\text { C } & 19.508777150 & -31.753171463 & 0.783608775\end{array}$

$\begin{array}{llll}\text { C } & 19.796538131 & -30.950825122 & -0.319011850\end{array}$

$\begin{array}{llll}\text { C } & 22.050710305 & -26.870833209 & -1.412437302\end{array}$

$\begin{array}{llll}\text { O } & 22.444657911 & -25.845346544 & -0.510605297\end{array}$

$\mathrm{H} \quad 16.143760718 \quad-23.869412583 \quad-1.106031351$

$\mathrm{H} \quad 15.633110764 \quad-26.156876111 \quad-2.027752841$

H $\quad 20.183515696 \quad-25.232091569 \quad-0.358517165$

$\begin{array}{llll}\mathrm{H} & 18.352105000 & -28.914179697 & -2.491032512\end{array}$

$\begin{array}{llll}\mathrm{H} & 20.048268680 & -29.465226241 & -2.484623197\end{array}$

$\begin{array}{llll}\mathrm{H} & 20.810277157 & -27.195602718 & 0.356465129\end{array}$

$\mathrm{H} \quad 21.307296386 \quad-28.696692128 \quad-0.446295229$

$\begin{array}{llll}\mathrm{H} & 19.455253040 & -23.001868895 & 0.234371306\end{array}$

$\mathrm{H} \quad 17.869642116 \quad-22.442411501 \quad-0.037213801$

H $\quad 17.125793891 \quad-28.844169069 \quad-0.173216726$

$\begin{array}{llll}\mathrm{H} & 16.622863965 & -30.270118979 & 1.800918558\end{array}$

$\mathrm{H} \quad 18.149661437 \quad-32.131213239 \quad 2.411718270$

H $\quad 20.173617890 \quad-32.572523146 \quad 1.046676520$

$\begin{array}{llll}\mathrm{H} & 20.684010714 & -31.150967014 & -0.914485303\end{array}$

$\mathrm{H} \quad 22.934450618 \quad-27.491531077 \quad-1.634028524$

$\begin{array}{llll}\mathrm{H} & 21.659653995 & -26.422336717 & -2.343124659\end{array}$

$\mathrm{H} \quad 22.999608045 \quad-25.184687806 \quad-0.963595814$

\section{Rotamer If}

01

$\begin{array}{llll}\mathrm{C} & 16.706665932 & -24.795993822 & -1.275215178 \\ \mathrm{C} & 17.978406876 & -24.502054001 & -0.793858155 \\ \mathrm{~N} & 18.986588486 & -25.388652411 & -0.743686787 \\ \mathrm{C} & 18.757507887 & -26.664979632 & -1.197731591\end{array}$




$\begin{array}{lrrr}\mathrm{C} & 17.497381048 & -27.064947458 & -1.687742362 \\ \mathrm{C} & 16.465750809 & -26.111803591 & -1.732046840 \\ \mathrm{~N} & 19.861218387 & -27.501382829 & -1.204421922 \\ \mathrm{C} & 19.711598997 & -28.909005043 & -1.578077624 \\ \mathrm{C} & 21.023831533 & -27.195842502 & -0.351109195 \\ \mathrm{~N} & 15.224066989 & -26.463199236 & -2.205755940 \\ \mathrm{C} & 19.141698426 & -29.816874941 & -0.515388682 \\ \mathrm{C} & 18.533604681 & -29.333376815 & 0.640917130 \\ \mathrm{C} & 18.012753849 & -30.221901340 & 1.580548088 \\ \mathrm{C} & 18.097539844 & -31.595505282 & 1.367800048 \\ \mathrm{C} & 18.704171532 & -32.081288287 & 0.209748826 \\ \mathrm{C} & 19.224592879 & -31.196160045 & -0.729615727 \\ \mathrm{C} & 22.072118200 & -26.397011358 & -1.156220198 \\ \mathrm{O} & 21.963212651 & -25.020056102 & -0.885779312 \\ \mathrm{H} & 15.932304527 & -24.044797422 & -1.294953101 \\ \mathrm{H} & 18.229259577 & -23.499677527 & -0.418069711 \\ \mathrm{H} & 17.314309195 & -28.080809740 & -2.003032665 \\ \mathrm{H} & 19.079071104 & -28.975142481 & -2.500431367 \\ \mathrm{H} & 20.721261445 & -29.280441599 & -1.891221501 \\ \mathrm{H} & 20.716124262 & -26.593264989 & 0.539654018 \\ \mathrm{H} & 21.460982521 & -28.134974602 & 0.047150792 \\ \mathrm{H} & 15.041920067 & -27.382064741 & -2.540042835 \\ \mathrm{H} & 14.486525384 & -25.798460201 & -2.255519245 \\ \mathrm{H} & 18.453324207 & -28.260762519 & 0.818528708 \\ \mathrm{H} & 17.537283077 & -29.837324692 & 2.481582011 \\ \mathrm{H} & 17.691383373 & -32.288143880 & 2.101255197 \\ \mathrm{H} & 18.770645355 & -33.154138810 & 0.040658298 \\ \mathrm{H} & 19.695157425 & -31.582278122 & -1.630986561 \\ \mathrm{H} & 23.102461167 & -26.643089106 & -0.844638843 \\ \mathrm{H} & 21.960749228 & -26.550162633 & -2.244893329 \\ \mathrm{H} & 21.006089316 & -24.763287289 & -0.876475704 \\ & & & \end{array}$

\section{Rotamer Ig}

01

$\begin{array}{lrrr}\mathrm{C} & 16.579553224 & -24.834299287 & -1.574332384 \\ \mathrm{C} & 16.343413593 & -26.169832829 & -1.891240556 \\ \mathrm{~N} & 17.290209849 & -27.120870938 & -1.888970098 \\ \mathrm{C} & 18.577159397 & -26.760873036 & -1.574898212 \\ \mathrm{C} & 18.916988885 & -25.437207302 & -1.227423683 \\ \mathrm{C} & 17.899443299 & -24.466569942 & -1.233454426 \\ \mathrm{~N} & 19.533633651 & -27.757443718 & -1.719988656 \\ \mathrm{C} & 19.103216826 & -29.165336637 & -1.819710347 \\ \mathrm{C} & 20.865449354 & -27.562703298 & -1.124276036 \\ \mathrm{~N} & 18.190928657 & -23.161835793 & -0.908305450 \\ \mathrm{C} & 18.956246642 & -29.820051431 & -0.470095831 \\ \mathrm{C} & 17.856793096 & -29.516136736 & 0.333765251 \\ \mathrm{C} & 17.731428550 & -30.099807010 & 1.591530852 \\ \mathrm{C} & 18.699368467 & -30.995043647 & 2.045226180 \\ \mathrm{C} & 19.790250064 & -31.309256451 & 1.236985583 \\ \mathrm{C} & 19.920000788 & -30.723313038 & -0.020731325\end{array}$




$\begin{array}{llll}\mathrm{C} & 21.791108360 & -26.911168032 & -2.175223942 \\ \mathrm{O} & 21.946991164 & -27.768747604 & -3.283875085 \\ \mathrm{H} & 15.780195420 & -24.110194730 & -1.588812027 \\ \mathrm{H} & 15.337968386 & -26.522911862 & -2.160500132 \\ \mathrm{H} & 19.927066340 & -25.163311138 & -0.968527706 \\ \mathrm{H} & 18.132777318 & -29.216631742 & -2.381482440 \\ \mathrm{H} & 19.851103981 & -29.699076405 & -2.452833951 \\ \mathrm{H} & 20.824557299 & -26.950560730 & -0.197893755 \\ \mathrm{H} & 21.294201128 & -28.546007905 & -0.819575287 \\ \mathrm{H} & 19.112333141 & -22.886762376 & -0.656436256 \\ \mathrm{H} & 17.481739629 & -22.465268624 & -0.898855603 \\ \mathrm{H} & 17.097666017 & -28.818924406 & -0.028756379 \\ \mathrm{H} & 16.875860524 & -29.857853740 & 2.218877857 \\ \mathrm{H} & 18.601151304 & -31.450426139 & 3.027950726 \\ \mathrm{H} & 20.541414334 & -32.013543489 & 1.587472966 \\ \mathrm{H} & 20.771636607 & -30.976113537 & -0.649164116 \\ \mathrm{H} & 21.400854346 & -25.939694335 & -2.526811341 \\ \mathrm{H} & 22.819530639 & -26.790577438 & -1.790591893 \\ \mathrm{H} & 21.060611262 & -27.972132583 & -3.657653456\end{array}$

\section{Rotamer II}

01

$\begin{array}{lrrr}\mathrm{C} & 3.384922000 & -2.002774000 & 0.205755000 \\ \mathrm{C} & 2.218979000 & -2.266164000 & 0.905308000 \\ \mathrm{~N} & 1.164348000 & -1.446934000 & 0.979943000 \\ \mathrm{C} & 1.243545000 & -0.265013000 & 0.333073000 \\ \mathrm{C} & 2.392654000 & 0.111907000 & -0.395695000 \\ \mathrm{C} & 3.474670000 & -0.770011000 & -0.471568000 \\ \mathrm{~N} & 0.138530000 & 0.563660000 & 0.424659000 \\ \mathrm{C} & -1.008983000 & 0.203090000 & 1.263437000 \\ \mathrm{C} & 0.034414000 & 1.798514000 & -0.333575000 \\ \mathrm{~N} & 4.592666000 & -0.459151000 & -1.227550000 \\ \mathrm{C} & -2.210614000 & -0.297673000 & 0.480274000 \\ \mathrm{C} & -2.132815000 & -1.492927000 & -0.250654000 \\ \mathrm{C} & -3.234214000 & -1.956342000 & -0.967002000 \\ \mathrm{C} & -4.431477000 & -1.234153000 & -0.965842000 \\ \mathrm{C} & -4.519621000 & -0.046608000 & -0.241510000 \\ \mathrm{C} & -3.413307000 & 0.416635000 & 0.475761000 \\ \mathrm{C} & 0.633625000 & 3.004470000 & 0.396120000 \\ \mathrm{O} & 0.454994000 & 4.132842000 & -0.450795000 \\ \mathrm{H} & 4.196650000 & -2.722405000 & 0.176861000 \\ \mathrm{H} & 2.117690000 & -3.207391000 & 1.444364000 \\ \mathrm{H} & 2.457889000 & 1.071845000 & -0.893662000 \\ \mathrm{H} & -0.666309000 & -0.564997000 & 1.957287000 \\ \mathrm{H} & -1.301233000 & 1.084055000 & 1.848857000 \\ \mathrm{H} & 0.512843000 & 1.693135000 & -1.312115000 \\ \mathrm{H} & -1.023356000 & 1.997249000 & -0.531550000 \\ \mathrm{H} & 4.736123000 & 0.517771000 & -1.438744000 \\ \mathrm{H} & 5.444086000 & -0.944359000 & -0.985295000 \\ \mathrm{H} & -1.203886000 & -2.055245000 & -0.241748000\end{array}$




$\begin{array}{rrrr}\mathrm{H} & -3.161902000 & -2.885410000 & -1.525715000 \\ \mathrm{H} & -5.288633000 & -1.597491000 & -1.525558000 \\ \mathrm{H} & -5.445465000 & 0.521798000 & -0.234650000 \\ \mathrm{H} & -3.487548000 & 1.342115000 & 1.042720000 \\ \mathrm{H} & 0.123630000 & 3.135543000 & 1.363493000 \\ \mathrm{H} & 1.697042000 & 2.818831000 & 0.610959000 \\ \mathrm{H} & 0.792960000 & 4.911479000 & 0.008221000\end{array}$

\section{Rotamer III}

01

$\begin{array}{llll}\text { C } & 3.385810000 & -2.001804000 & 0.206009000\end{array}$

$\begin{array}{llll}\text { C } & 2.220025000 & -2.265599000 & 0.905665000\end{array}$

$\begin{array}{llll}\mathrm{N} & 1.165191000 & -1.446667000 & 0.980543000\end{array}$

$\begin{array}{llll}\text { C } & 1.243969000 & -0.264615000 & 0.333863000\end{array}$

$\begin{array}{llll}\text { C } & 2.392971000 & 0.112798000 & -0.394841000\end{array}$

$\begin{array}{llll}\text { C } & 3.475175000 & -0.768879000 & -0.471063000\end{array}$

$\begin{array}{llll}\mathrm{N} & 0.138670000 & 0.563662000 & 0.425607000\end{array}$

$\begin{array}{llll}\text { C } & -1.008882000 & 0.202220000 & 1.263930000\end{array}$

$\begin{array}{llll}\text { C } & 0.033444000 & 1.797847000 & -0.333539000\end{array}$

$\begin{array}{llll}\mathrm{N} & 4.592980000 & -0.457615000 & -1.227172000\end{array}$

$\begin{array}{llll}\text { C } & -2.210217000 & -0.298458000 & 0.480275000\end{array}$

$\begin{array}{llll}\text { C } & -2.130796000 & -1.491257000 & -0.254488000\end{array}$

C $\quad-3.232011000 \quad-1.954584000 \quad-0.971176000$

C $\quad-4.430722000 \quad-1.234828000 \quad-0.966488000$

$\begin{array}{llll}\text { C } & -4.520501000 & -0.049777000 & -0.238284000\end{array}$

$\begin{array}{llll}\text { C } & -3.414353000 & 0.413428000 & 0.479260000\end{array}$

$\begin{array}{llll}\text { C } & 0.631199000 & 3.004969000 & 0.395411000\end{array}$

$\begin{array}{llll}\mathrm{O} & 0.451951000 & 4.132421000 & -0.452593000\end{array}$

$\mathrm{H} \quad 4.197669000 \quad-2.721271000 \quad 0.176843000$

$\begin{array}{llll}\mathrm{H} & 2.119036000 & -3.206945000 & 1.444572000\end{array}$

$\mathrm{H} \quad 2.458052000 \quad 1.072835000 \quad-0.892647000$

$\begin{array}{llll}\mathrm{H} & -0.666035000 & -0.566214000 & 1.957327000\end{array}$

$\begin{array}{llll}\mathrm{H} & -1.301531000 & 1.082684000 & 1.849915000\end{array}$

$\begin{array}{llll}\mathrm{H} & 0.512364000 & 1.692348000 & -1.311819000\end{array}$

$\mathrm{H} \quad-1.024491000 \quad 1.995217000 \quad-0.532016000$

$\mathrm{H} \quad 4.736188000 \quad 0.519373000 \quad-1.438237000$

$\mathrm{H} \quad 5.444558000 \quad-0.942678000 \quad-0.985183000$

$\mathrm{H} \quad-1.200786000 \quad-2.051826000 \quad-0.248310000$

$\mathrm{H} \quad-3.158409000 \quad-2.881723000 \quad-1.532920000$

$\mathrm{H} \quad-5.287713000 \quad-1.598108000 \quad-1.526489000$

$\begin{array}{llll}\mathrm{H} & -5.447455000 & 0.516779000 & -0.228689000\end{array}$

$\begin{array}{llll}\mathrm{H} & -3.489823000 & 1.337034000 & 1.049107000\end{array}$

$\mathrm{H} \quad 0.120697000 \quad 3.136479000 \quad 1.362461000$

$\begin{array}{llll}\mathrm{H} & 1.694687000 & 2.820409000 & 0.610852000\end{array}$

$\mathrm{H} \quad 0.788830000 \quad 4.911813000 \quad 0.005938000$

\section{Rotamer IV}

01

$\begin{array}{llll}\text { C } & 3.385459000 & -2.002689000 & 0.206087000\end{array}$

$\begin{array}{llll}\text { C } & 2.219143000 & -2.266335000 & 0.904912000\end{array}$ 


$\begin{array}{lrrr}\mathrm{N} & 1.164324000 & -1.447289000 & 0.978992000 \\ \mathrm{C} & 1.243728000 & -0.265274000 & 0.332328000 \\ \mathrm{C} & 2.393256000 & 0.111944000 & -0.395659000 \\ \mathrm{C} & 3.475404000 & -0.769844000 & -0.471070000 \\ \mathrm{~N} & 0.138483000 & 0.563142000 & 0.423243000 \\ \mathrm{C} & -1.008794000 & 0.203071000 & 1.262528000 \\ \mathrm{C} & 0.034858000 & 1.798401000 & -0.334393000 \\ \mathrm{~N} & 4.593771000 & -0.458750000 & -1.226420000 \\ \mathrm{C} & -2.210818000 & -0.297527000 & 0.479921000 \\ \mathrm{C} & -2.133461000 & -1.492833000 & -0.251009000 \\ \mathrm{C} & -3.235275000 & -1.956191000 & -0.966751000 \\ \mathrm{C} & -4.432504000 & -1.233942000 & -0.964954000 \\ \mathrm{C} & -4.520226000 & -0.046395000 & -0.240584000 \\ \mathrm{C} & -3.413489000 & 0.416815000 & 0.476066000 \\ \mathrm{C} & 0.633393000 & 3.004090000 & 0.396301000 \\ \mathrm{O} & 0.454847000 & 4.132873000 & -0.450085000 \\ \mathrm{H} & 4.197302000 & -2.722201000 & 0.177576000 \\ \mathrm{H} & 2.117693000 & -3.207626000 & 1.443825000 \\ \mathrm{H} & 2.458682000 & 1.071985000 & -0.893405000 \\ \mathrm{H} & -0.666082000 & -0.565009000 & 1.956369000 \\ \mathrm{H} & -1.300567000 & 1.084191000 & 1.847971000 \\ \mathrm{H} & 0.514126000 & 1.693530000 & -1.312560000 \\ \mathrm{H} & -1.022775000 & 1.997147000 & -0.533121000 \\ \mathrm{H} & 4.737115000 & 0.518194000 & -1.437590000 \\ \mathrm{H} & 5.445174000 & -0.943747000 & -0.983685000 \\ \mathrm{H} & -1.204552000 & -2.055209000 & -0.242581000 \\ \mathrm{H} & -3.163334000 & -2.885279000 & -1.525481000 \\ \mathrm{H} & -5.289973000 & -1.597271000 & -1.524197000 \\ \mathrm{H} & -5.446050000 & 0.522037000 & -0.233218000 \\ \mathrm{H} & -3.487405000 & 1.342283000 & 1.043092000 \\ \mathrm{H} & 0.122917000 & 3.134495000 & 1.363506000 \\ \mathrm{H} & 1.696761000 & 2.818633000 & 0.611518000 \\ \mathrm{H} & 0.792106000 & 4.911411000 & 0.009618000\end{array}$

\section{Rotamer V}

$\begin{array}{lrrr}11 & & & \\ \mathrm{C} & -1.845934000 & -2.967832000 & -0.234768000 \\ \mathrm{C} & -0.597039000 & -2.634348000 & -0.648891000 \\ \mathrm{~N} & -0.152052000 & -1.343738000 & -0.606585000 \\ \mathrm{C} & -0.924810000 & -0.308595000 & -0.133477000 \\ \mathrm{C} & -2.220998000 & -0.616811000 & 0.303975000 \\ \mathrm{C} & -2.700084000 & -1.934854000 & 0.269094000 \\ \mathrm{~N} & -0.378656000 & 0.934156000 & -0.123612000 \\ \mathrm{C} & 0.874392000 & 1.222339000 & -0.851660000 \\ \mathrm{C} & -1.116048000 & 2.069670000 & 0.444268000 \\ \mathrm{~N} & -3.944687000 & -2.240110000 & 0.684866000 \\ \mathrm{C} & 2.124202000 & 0.569058000 & -0.277236000 \\ \mathrm{C} & 2.374445000 & 0.562036000 & 1.102519000 \\ \mathrm{C} & 3.547367000 & -0.001610000 & 1.600692000 \\ \mathrm{C} & 4.483646000 & -0.565214000 & 0.728830000\end{array}$




$\begin{array}{rrrr}\mathrm{C} & 4.242191000 & -0.564913000 & -0.644229000 \\ \mathrm{C} & 3.065302000 & -0.001964000 & -1.145785000 \\ \mathrm{C} & -2.052184000 & 2.763126000 & -0.552546000 \\ \mathrm{O} & -2.764170000 & 3.716913000 & 0.207831000 \\ \mathrm{H} & -2.180028000 & -3.996786000 & -0.284567000 \\ \mathrm{H} & 0.111515000 & -3.359762000 & -1.028955000 \\ \mathrm{H} & -2.870855000 & 0.174332000 & 0.649725000 \\ \mathrm{H} & 0.759391000 & 0.938441000 & -1.905840000 \\ \mathrm{H} & 0.984335000 & 2.309639000 & -0.839273000 \\ \mathrm{H} & -1.692617000 & 1.739231000 & 1.311251000 \\ \mathrm{H} & -0.387518000 & 2.792418000 & 0.818324000 \\ \mathrm{H} & -4.563441000 & -1.536184000 & 1.055781000 \\ \mathrm{H} & -4.289145000 & -3.186426000 & 0.651807000 \\ \mathrm{H} & 1.652122000 & 0.997742000 & 1.787085000 \\ \mathrm{H} & 3.734383000 & 0.001681000 & 2.669904000 \\ \mathrm{H} & 5.397031000 & -1.001082000 & 1.120666000 \\ \mathrm{H} & 4.966366000 & -0.997662000 & -1.327085000 \\ \mathrm{H} & 2.889282000 & 0.010973000 & -2.219382000 \\ \mathrm{H} & -1.460560000 & 3.224617000 & -1.357797000 \\ \mathrm{H} & -2.717993000 & 2.020913000 & -1.021819000 \\ \mathrm{H} & -3.246704000 & 4.304196000 & -0.387614000 \\ \mathrm{H} & 0.828874000 & -1.164276000 & -0.802266000\end{array}$

\section{Rotamer VI}

\begin{tabular}{lrrr}
11 & & & \\
$\mathrm{C}$ & -1.841000000 & -2.968823000 & -0.235845000 \\
$\mathrm{C}$ & -0.592095000 & -2.633271000 & -0.648280000 \\
$\mathrm{~N}$ & -0.148972000 & -1.342084000 & -0.604498000 \\
$\mathrm{C}$ & -0.923699000 & -0.308369000 & -0.131496000 \\
$\mathrm{C}$ & -2.219983000 & -0.618734000 & 0.304169000 \\
$\mathrm{C}$ & -2.697183000 & -1.937423000 & 0.267728000 \\
$\mathrm{~N}$ & -0.379214000 & 0.935184000 & -0.119881000 \\
$\mathrm{C}$ & 0.873379000 & 1.226189000 & -0.847707000 \\
$\mathrm{C}$ & -1.120279000 & 2.069391000 & 0.445928000 \\
$\mathrm{~N}$ & -3.941886000 & -2.244665000 & 0.681743000 \\
$\mathrm{C}$ & 2.123954000 & 0.572230000 & -0.275807000 \\
$\mathrm{C}$ & 2.375720000 & 0.562569000 & 1.103668000 \\
$\mathrm{C}$ & 3.549372000 & -0.001710000 & 1.599408000 \\
$\mathrm{C}$ & 4.484867000 & -0.563321000 & 0.725411000 \\
$\mathrm{C}$ & 4.241870000 & -0.560448000 & -0.647374000 \\
$\mathrm{C}$ & 3.064269000 & 0.003165000 & -1.146506000 \\
$\mathrm{C}$ & -2.057029000 & 2.759397000 & -0.552700000 \\
$\mathrm{O}$ & -2.773846000 & 3.710745000 & 0.206213000 \\
$\mathrm{H}$ & -2.173589000 & -3.998205000 & -0.286793000 \\
$\mathrm{H}$ & 0.117923000 & -3.357403000 & -1.028057000 \\
$\mathrm{H}$ & -2.871394000 & 0.171191000 & 0.649738000 \\
$\mathrm{H}$ & 0.758184000 & 0.945222000 & -1.902669000 \\
$\mathrm{H}$ & 0.982708000 & 2.313504000 & -0.832148000 \\
$\mathrm{H}$ & -1.697202000 & 1.738270000 & 1.312426000 \\
$\mathrm{H}$ & -0.394104000 & 2.794286000 & 0.820358000 \\
& & & \\
\hline
\end{tabular}




$\begin{array}{rrrr}\mathrm{H} & -4.562147000 & -1.541797000 & 1.052151000 \\ \mathrm{H} & -4.285084000 & -3.191397000 & 0.647480000 \\ \mathrm{H} & 1.653995000 & 0.996686000 & 1.789873000 \\ \mathrm{H} & 3.737570000 & -0.000471000 & 2.668417000 \\ \mathrm{H} & 5.398810000 & -0.999677000 & 1.115400000 \\ \mathrm{H} & 4.965403000 & -0.991649000 & -1.331888000 \\ \mathrm{H} & 2.887012000 & 0.018136000 & -2.219868000 \\ \mathrm{H} & -1.465697000 & 3.222915000 & -1.356989000 \\ \mathrm{H} & -2.719301000 & 2.014634000 & -1.022926000 \\ \mathrm{H} & -3.256713000 & 4.296730000 & -0.390238000 \\ \mathrm{H} & 0.831921000 & -1.161038000 & -0.799104000\end{array}$

\section{Rotamer VII}

11

C $\quad-1.846126000 \quad-2.967533000$

$\begin{array}{lll}\text { C } & -0.596868000 & -2.634074000\end{array}$

$-0.235205000$

$\mathrm{N} \quad-0.151766000 \quad-1.343550000$

$-0.648270000$

C $\quad-0.924771000$

$-0.308438000$

$-0.605207000$

C $\quad-2.221311000$

$-0.132420000$

C $\quad-2.700539000$

$-0.616616000$

0.303994000

$\mathrm{N} \quad-0.378416000$

$-1.934607000$

0.268319000

C $\quad 0.874412000$

0.934251000

$-0.121929000$

1.222614000

$-0.850248000$

C -1.116433000

2.069834000

0.445017000

$\mathrm{N} \quad-3.945525000$

$-2.239830000$

0.682953000

C 2.124439000

0.568967000

$-0.276718000$

C $\quad 2.374906000$

0.560189000

1.103000000

C $\quad 3.548294000$

$-0.003355000$

1.600193000

C $\quad 4.484869000$

$-0.565016000$

0.727409000

C $\quad 4.243189000$

$-0.562981000$

$-0.645614000$

C $\quad 3.065780000$

$-0.000240000$

$-1.146186000$

C $\quad-2.052512000$

2.762379000

$-0.552506000$

O -2.765976000

3.715533000

0.207292000

$\mathrm{H} \quad-2.180317000$

$-3.996425000$

$-0.285613000$

$\mathrm{H} \quad 0.111884000$

$-3.359461000$

$-1.028017000$

$\mathrm{H} \quad-2.871392000$

0.174496000

0.649402000

H 0.759009000

0.939334000

$-1.904563000$

$\mathrm{H} \quad 0.984554000$

2.309889000

$-0.837299000$

$\mathrm{H} \quad-1.693324000$

1.739691000

1.311904000

$\mathrm{H} \quad-0.388300000$

2.793004000

0.819027000

$\mathrm{H} \quad-4.564576000$

$-1.535914000$

1.053390000

$\mathrm{H} \quad-4.290142000$

$-3.186062000$

0.649124000

$\mathrm{H} \quad 1.652431000$

0.994496000

1.788290000

$\mathrm{H} \quad 3.735452000$

$-0.001438000$

2.669385000

$\mathrm{H} \quad 5.398633000$

$-1.000752000$

1.118504000

$\mathrm{H} \quad 4.967562000$

$-0.994250000$

$-1.329193000$

$\mathrm{H} \quad 2.889533000$

0.014010000

$-2.219724000$

$\mathrm{H} \quad-1.460871000$

3.224402000

$-1.357435000$

$\mathrm{H} \quad-2.717271000$

2.019432000

$-1.022069000$

H $\quad-3.248379000$

4.302503000

$-0.388567000$

$\mathrm{H}$

$-1.164050000$

$-0.800185000$ 


$\begin{array}{lrrr}\text { Rotamer VIII } & & \\ 11 & & & \\ \text { C } & -1.842199000 & -2.968364000 & -0.237017000 \\ \text { C } & -0.593526000 & -2.633002000 & -0.650329000 \\ \text { N } & -0.149971000 & -1.341973000 & -0.606355000 \\ \text { C } & -0.923946000 & -0.308230000 & -0.132192000 \\ \text { C } & -2.219954000 & -0.618401000 & 0.304418000 \\ \text { C } & -2.697648000 & -1.936910000 & 0.267728000 \\ \text { N } & -0.379019000 & 0.935098000 & -0.120331000 \\ \mathrm{C} & 0.873781000 & 1.225676000 & -0.847873000 \\ \mathrm{C} & -1.119458000 & 2.069475000 & 0.445961000 \\ \mathrm{~N} & -3.942076000 & -2.243943000 & 0.682702000 \\ \mathrm{C} & 2.124026000 & 0.571390000 & -0.275565000 \\ \mathrm{C} & 2.374223000 & 0.559552000 & 1.104174000 \\ \mathrm{C} & 3.547776000 & -0.004614000 & 1.600284000 \\ \mathrm{C} & 4.484751000 & -0.563892000 & 0.726396000 \\ \mathrm{C} & 4.243302000 & -0.558862000 & -0.646661000 \\ \mathrm{C} & 3.065760000 & 0.004539000 & -1.146159000 \\ \mathrm{C} & -2.056290000 & 2.759959000 & -0.552264000 \\ \mathrm{O} & -2.772012000 & 3.711961000 & 0.206848000 \\ \mathrm{H} & -2.175141000 & -3.997626000 & -0.288132000 \\ \mathrm{H} & 0.115952000 & -3.357189000 & -1.031006000 \\ \mathrm{H} & -2.870685000 & 0.171534000 & 0.651247000 \\ \mathrm{H} & 0.758832000 & 0.944683000 & -1.902858000 \\ \mathrm{H} & 0.983427000 & 2.312973000 & -0.832458000 \\ \mathrm{H} & -1.696079000 & 1.738543000 & 1.312733000 \\ \mathrm{H} & -0.392845000 & 2.794082000 & 0.820100000 \\ \mathrm{H} & -4.561740000 & -1.541054000 & 1.054066000 \\ \mathrm{H} & -4.285502000 & -3.190595000 & 0.648551000 \\ \mathrm{H} & 1.651328000 & 0.991835000 & 1.790297000 \\ \mathrm{H} & 3.734735000 & -0.005072000 & 2.669512000 \\ \mathrm{H} & 5.398630000 & -1.000127000 & 1.116668000 \\ \mathrm{H} & 4.967957000 & -0.988302000 & -1.331094000 \\ \mathrm{H} & 2.889686000 & 0.021109000 & -2.219690000 \\ \mathrm{H} & -1.464983000 & 3.222856000 & -1.356931000 \\ \mathrm{H} & -2.719364000 & 2.015643000 & -1.022050000 \\ \mathrm{H} & -3.255006000 & 4.297996000 & -0.389452000 \\ \mathrm{H} & 0.830772000 & -1.161161000 & -0.801809000\end{array}$

\section{Rotamer IX}

$\begin{array}{lrrr}11 & & & \\ \mathrm{C} & 4.151678000 & -0.322601000 & 0.788067000 \\ \mathrm{C} & 3.229255000 & 0.390802000 & 1.522591000 \\ \mathrm{~N} & 1.928792000 & 0.492446000 & 1.194622000 \\ \mathrm{C} & 1.570164000 & -0.144657000 & 0.103316000 \\ \mathrm{C} & 2.377815000 & -0.894711000 & -0.725308000 \\ \mathrm{C} & 3.741733000 & -0.996812000 & -0.379401000 \\ \mathrm{~N} & 0.123137000 & -0.020376000 & -0.240486000 \\ \mathrm{C} & -0.789709000 & -0.319010000 & 0.970679000\end{array}$




$\begin{array}{lrrr}\mathrm{C} & -0.210862000 & 1.266346000 & -0.977744000 \\ \mathrm{~N} & 4.610845000 & -1.710565000 & -1.130731000 \\ \mathrm{C} & -2.202851000 & -0.580466000 & 0.540442000 \\ \mathrm{C} & -3.185403000 & 0.403812000 & 0.665928000 \\ \mathrm{C} & -4.490743000 & 0.148309000 & 0.260619000 \\ \mathrm{C} & -4.823808000 & -1.091187000 & -0.275771000 \\ \mathrm{C} & -3.853280000 & -2.081731000 & -0.397155000 \\ \mathrm{C} & -2.550522000 & -1.829153000 & 0.014567000 \\ \mathrm{C} & 0.093577000 & 2.556323000 & -0.222982000 \\ \mathrm{O} & -0.524086000 & 3.551922000 & -1.023923000 \\ \mathrm{H} & 5.182479000 & -0.364532000 & 1.110937000 \\ \mathrm{H} & 3.533319000 & 0.910182000 & 2.421651000 \\ \mathrm{H} & 1.992989000 & -1.387953000 & -1.608497000 \\ \mathrm{H} & -0.340981000 & -1.189213000 & 1.443077000 \\ \mathrm{H} & -0.690151000 & 0.521482000 & 1.645639000 \\ \mathrm{H} & 0.346138000 & 1.239837000 & -1.911508000 \\ \mathrm{H} & -1.273364000 & 1.222890000 & -1.204344000 \\ \mathrm{H} & 4.319433000 & -2.186910000 & -1.964317000 \\ \mathrm{H} & 5.579157000 & -1.778123000 & -0.875277000 \\ \mathrm{H} & -2.936396000 & 1.367205000 & 1.092023000 \\ \mathrm{H} & -5.246200000 & 0.913915000 & 0.367948000 \\ \mathrm{H} & -5.839656000 & -1.289707000 & -0.587758000 \\ \mathrm{H} & -4.114638000 & -3.052110000 & -0.795359000 \\ \mathrm{H} & -1.810129000 & -2.618946000 & -0.048076000 \\ \mathrm{H} & -0.327091000 & 2.538025000 & 0.785179000 \\ \mathrm{H} & 1.168173000 & 2.715058000 & -0.135716000 \\ \mathrm{H} & -0.249051000 & 4.424078000 & -0.727257000 \\ \mathrm{H} & -0.083025000 & -0.762688000 & -0.907899000\end{array}$

\section{Rotamer X}

11

$\begin{array}{llll}\text { C } & 17.191741000 & -25.638229000 & -3.339708000\end{array}$

$\begin{array}{llll}\text { C } & 17.666123000 & -26.924229000 & -3.156082000\end{array}$

$\begin{array}{llll}\mathrm{N} & 18.400295000 & -27.310990000 & -2.093047000\end{array}$

$\begin{array}{llll}\text { C } & 18.649179000 & -26.365028000 & -1.207220000\end{array}$

C $\quad 18.243509000 \quad-25.043165000 \quad-1.253498000$

$\begin{array}{llll}\text { C } & 17.475318000 & -24.643481000 & -2.374300000\end{array}$

N $\quad 19.459528000 \quad-26.821820000 \quad-0.038948000$

C $\quad 18.883401000 \quad-28.112917000 \quad 0.595854000$

C $\quad 20.952812000 \quad-26.874231000 \quad-0.329089000$

$\begin{array}{llll}\mathrm{N} & 17.031560000 & -23.372777000 & -2.511330000\end{array}$

C $\quad 19.467862000 \quad-28.359030000 \quad 1.957167000$

$\begin{array}{llll}\text { C } & 20.510431000 & -29.278570000 & 2.139604000\end{array}$

C $\quad 21.047585000 \quad-29.489303000 \quad 3.409505000$

$\begin{array}{llll}\text { C } & 20.548901000 & -28.782909000 & 4.505360000\end{array}$

$\begin{array}{llll}\text { C } & 19.505504000 & -27.870059000 & 4.334627000\end{array}$

C $\quad 18.964365000 \quad-27.662513000 \quad 3.067147000$

C $\quad 21.379200000 \quad-27.824811000 \quad-1.448710000$

$\begin{array}{llll}\text { O } & 22.790604000 & -27.810475000 & -1.349186000\end{array}$

$\mathrm{H} \quad 16.603936000 \quad-25.396521000 \quad-4.218942000$ 


$\begin{array}{lrrr}\mathrm{H} & 17.452673000 & -27.694747000 & -3.891090000 \\ \mathrm{H} & 18.497895000 & -24.331546000 & -0.473200000 \\ \mathrm{H} & 17.806783000 & -27.937315000 & 0.636692000 \\ \mathrm{H} & 19.070440000 & -28.911686000 & -0.117711000 \\ \mathrm{H} & 21.257157000 & -25.854121000 & -0.571240000 \\ \mathrm{H} & 21.437762000 & -27.175935000 & 0.600806000 \\ \mathrm{H} & 17.237814000 & -22.663078000 & -1.827551000 \\ \mathrm{H} & 16.485691000 & -23.098279000 & -3.312084000 \\ \mathrm{H} & 20.894506000 & -29.839492000 & 1.291756000 \\ \mathrm{H} & 21.850010000 & -30.207392000 & 3.543080000 \\ \mathrm{H} & 20.965295000 & -28.950689000 & 5.493412000 \\ \mathrm{H} & 19.105301000 & -27.333408000 & 5.188680000 \\ \mathrm{H} & 18.129047000 & -26.975101000 & 2.946218000 \\ \mathrm{H} & 20.963952000 & -28.830814000 & -1.295072000 \\ \mathrm{H} & 21.020628000 & -27.466979000 & -2.420085000 \\ \mathrm{H} & 23.164432000 & -28.250122000 & -2.123465000 \\ \mathrm{H} & 19.359631000 & -26.110610000 & 0.690885000\end{array}$

\section{Rotamer XI}

11

C $\quad 18.655013000 \quad-23.633293000 \quad-2.215946000$

$\begin{array}{llll}\text { C } & 19.695381000 & -24.462747000 & -2.592995000\end{array}$

$\begin{array}{llll}\mathrm{N} & 19.858246000 & -25.721036000 & -2.136024000\end{array}$

C $\quad 18.940228000 \quad-26.132568000 \quad-1.282003000$

C $\quad 17.848679000 \quad-25.422211000 \quad-0.815152000$

C $\quad 17.685731000 \quad-24.100380000 \quad-1.297098000$

N $\quad 19.142655000 \quad-27.530068000 \quad-0.795900000$

$\begin{array}{llll}\text { C } & 19.372884000 & -28.521527000 & -1.964549000\end{array}$

$\begin{array}{llll}\text { C } & 20.164109000 & -27.631649000 & 0.328016000\end{array}$

N $\quad 16.650560000 \quad-23.324957000 \quad-0.900211000$

C $\quad 19.218000000 \quad-29.944817000 \quad-1.511149000$

C $\quad 20.338775000 \quad-30.736514000 \quad-1.223410000$

C $\quad 20.177361000 \quad-32.054245000 \quad-0.795496000$

$\begin{array}{llll}\text { C } & 18.896582000 & -32.590130000 & -0.650594000\end{array}$

C $\quad 17.774155000 \quad-31.811393000 \quad-0.941907000$

C $\quad 17.934271000 \quad-30.496306000 \quad-1.374696000$

$\begin{array}{llll}\text { C } & 21.580803000 & -27.170872000 & -0.017736000\end{array}$

$\begin{array}{llll}\text { O } & 22.322962000 & -27.563470000 & 1.121234000\end{array}$

$\mathrm{H} \quad 18.583622000 \quad-22.631164000 \quad-2.625374000$

H $\quad 20.443562000 \quad-24.113462000 \quad-3.298487000$

$\mathrm{H} \quad 17.141280000 \quad-25.845827000 \quad-0.107960000$

$\mathrm{H} \quad 18.622923000 \quad-28.243011000 \quad-2.707282000$

$\mathrm{H} \quad 20.354809000 \quad-28.290054000 \quad-2.370028000$

$\begin{array}{llll}\mathrm{H} & 19.772147000 & -27.039052000 & 1.156924000\end{array}$

$\begin{array}{llll}\mathrm{H} & 20.191813000 & -28.680994000 & 0.626358000\end{array}$

$\mathrm{H} \quad 15.963629000 \quad-23.654709000 \quad-0.242064000$

$\mathrm{H} \quad 16.543233000 \quad-22.386098000 \quad-1.248857000$

$\mathrm{H} \quad 21.338284000 \quad-30.327849000 \quad-1.345673000$

$\mathrm{H} \quad 21.050171000 \quad-32.662164000 \quad-0.580988000$

H $\quad 18.772648000 \quad-33.616723000 \quad-0.321160000$ 


$\begin{array}{lrrr}\mathrm{H} & 16.778626000 & -32.232571000 & -0.846564000 \\ \mathrm{H} & 17.057034000 & -29.905632000 & -1.632560000 \\ \mathrm{H} & 21.943263000 & -27.656097000 & -0.934939000 \\ \mathrm{H} & 21.607350000 & -26.088795000 & -0.186763000 \\ \mathrm{H} & 23.201983000 & -27.166273000 & 1.075424000 \\ \mathrm{H} & 18.259406000 & -27.831103000 & -0.374368000\end{array}$

\section{Rotamer XII}

11

C $\quad 18.689601000 \quad-23.411976000 \quad-1.644335000$

$\begin{array}{llll}\mathrm{C} & 19.847847000 & -24.092446000 & -1.972945000\end{array}$

N $\quad 20.027958000 \quad-25.413447000 \quad-1.770062000$

C $\quad 19.005061000 \quad-26.043197000 \quad-1.223690000$

$\begin{array}{llll}\text { C } & 17.790047000 & -25.500954000 & -0.844570000\end{array}$

$\begin{array}{llll}\text { C } & 17.609084000 & -24.112532000 & -1.058092000\end{array}$

$\begin{array}{llll}\mathrm{N} & 19.230107000 & -27.504659000 & -1.014528000\end{array}$

C $\quad 19.758019000 \quad-28.198518000 \quad-2.295832000$

$\begin{array}{llll}\text { C } & 20.028232000 & -27.813937000 & 0.244142000\end{array}$

$\begin{array}{llll}\mathrm{N} & 16.456745000 & -23.488951000 & -0.720976000\end{array}$

C $\quad 19.634581000 \quad-29.691975000 \quad-2.197342000$

C $\quad 20.738728000 \quad-30.486796000 \quad-1.858370000$

C $\quad 20.604860000 \quad-31.871923000 \quad-1.762993000$

C $\quad 19.368167000 \quad-32.472830000 \quad-2.004004000$

C $\quad 18.264197000 \quad-31.689897000 \quad-2.349527000$

$\begin{array}{llll}\text { C } & 18.397903000 & -30.306283000 & -2.450231000\end{array}$

$\begin{array}{llll}\text { C } & 21.439710000 & -27.227498000 & 0.296679000\end{array}$

$\begin{array}{llll}\text { O } & 21.987630000 & -27.836659000 & 1.450326000\end{array}$

$\mathrm{H} \quad 18.611670000 \quad-22.347330000 \quad-1.837842000$

$\mathrm{H} \quad 20.682129000 \quad-23.563504000 \quad-2.424266000$

$\mathrm{H} \quad 17.001657000 \quad-26.101017000 \quad-0.399212000$

H $\quad 19.143474000 \quad-27.787660000 \quad-3.099016000$

H $\quad 20.777338000 \quad-27.846297000 \quad-2.434021000$

$\mathrm{H} \quad 19.438855000 \quad-27.440787000 \quad 1.083955000$

H $\quad 20.089588000 \quad-28.901418000 \quad 0.311646000$

$\mathrm{H} \quad 15.689421000 \quad-23.985891000 \quad-0.299039000$

$\mathrm{H} \quad 16.337445000 \quad-22.501097000 \quad-0.877316000$

$\mathrm{H} \quad 21.706381000 \quad-30.025029000 \quad-1.681126000$

$\mathrm{H} \quad 21.465840000 \quad-32.480355000 \quad-1.506280000$

$\mathrm{H} \quad 19.266454000 \quad-33.550985000 \quad-1.933169000$

$\mathrm{H} \quad 17.306677000 \quad-32.157622000 \quad-2.554347000$

$\mathrm{H} \quad 17.542414000 \quad-29.705005000 \quad-2.752727000$

$\mathrm{H} \quad 22.007066000 \quad-27.476062000 \quad-0.611277000$

$\begin{array}{llll}\mathrm{H} & 21.404253000 & -26.135162000 & 0.372319000\end{array}$

$\begin{array}{llll}\mathrm{H} & 22.822250000 & -27.402550000 & 1.668277000\end{array}$

$\mathrm{H} \quad 18.312173000 \quad-27.928697000 \quad-0.853099000$

\section{Rotamer XIII}

11

$\begin{array}{llll}\text { C } & 1.918214000 & 3.083645000 & 0.447724000\end{array}$

$\begin{array}{llll}\text { C } & 2.647133000 & 1.958686000 & 0.854262000\end{array}$ 


$\begin{array}{lrrr}\mathrm{N} & 2.270134000 & 0.703346000 & 0.649471000 \\ \mathrm{C} & 1.106308000 & 0.444601000 & 0.009427000 \\ \mathrm{C} & 0.270786000 & 1.512976000 & -0.442731000 \\ \mathrm{C} & 0.723524000 & 2.788968000 & -0.195912000 \\ \mathrm{~N} & 0.762533000 & -0.855907000 & -0.173091000 \\ \mathrm{C} & -0.403712000 & -1.219911000 & -0.982661000 \\ \mathrm{C} & 1.604899000 & -1.933957000 & 0.366787000 \\ \mathrm{~N} & -0.140273000 & 3.928893000 & -0.644270000 \\ \mathrm{C} & -1.758429000 & -0.932843000 & -0.346874000 \\ \mathrm{C} & -1.950193000 & -0.999980000 & 1.038126000 \\ \mathrm{C} & -3.215185000 & -0.784376000 & 1.586564000 \\ \mathrm{C} & -4.302703000 & -0.500207000 & 0.758246000 \\ \mathrm{C} & -4.119416000 & -0.429992000 & -0.623614000 \\ \mathrm{C} & -2.852964000 & -0.641407000 & -1.171660000 \\ \mathrm{C} & 2.704808000 & -2.379595000 & -0.599475000 \\ \mathrm{O} & 3.341776000 & -3.474088000 & 0.030685000 \\ \mathrm{H} & 2.271875000 & 4.091189000 & 0.634103000 \\ \mathrm{H} & 3.592048000 & 2.093070000 & 1.375680000 \\ \mathrm{H} & -0.691229000 & 1.322299000 & -0.903029000 \\ \mathrm{H} & -0.343797000 & -0.723338000 & -1.960373000 \\ \mathrm{H} & -0.314677000 & -2.290639000 & -1.188001000 \\ \mathrm{H} & 2.063026000 & -1.598945000 & 1.296976000 \\ \mathrm{H} & 0.958705000 & -2.786734000 & 0.590317000 \\ \mathrm{H} & -1.054702000 & 3.592128000 & -0.965626000 \\ \mathrm{H} & -0.302827000 & 4.591354000 & 0.123351000 \\ \mathrm{H} & -1.110519000 & -1.222126000 & 1.690060000 \\ \mathrm{H} & -3.352232000 & -0.845308000 & 2.661906000 \\ \mathrm{H} & -5.286978000 & -0.339268000 & 1.186713000 \\ \mathrm{H} & -4.960855000 & -0.215304000 & -1.275478000 \\ \mathrm{H} & -2.718950000 & -0.597311000 & -2.250646000 \\ \mathrm{H} & 2.260563000 & -2.662266000 & -1.568419000 \\ \mathrm{H} & 3.392091000 & -1.539535000 & -0.776726000 \\ \mathrm{H} & 4.085340000 & -3.760233000 & -0.514237000 \\ \mathrm{H} & 0.293729000 & 4.449712000 & -1.416067000\end{array}$

\section{Rotamer XIV}

$\begin{array}{lrrr}11 & & & \\ \mathrm{C} & 1.914319000 & 3.085300000 & 0.448034000 \\ \mathrm{C} & 2.644282000 & 1.961203000 & 0.855031000 \\ \mathrm{~N} & 2.268786000 & 0.705420000 & 0.650162000 \\ \mathrm{C} & 1.105539000 & 0.445289000 & 0.009650000 \\ \mathrm{C} & 0.269080000 & 1.512706000 & -0.443097000 \\ \mathrm{C} & 0.720310000 & 2.789210000 & -0.196232000 \\ \mathrm{~N} & 0.763289000 & -0.855618000 & -0.172906000 \\ \mathrm{C} & -0.402440000 & -1.220936000 & -0.982615000 \\ \mathrm{C} & 1.607080000 & -1.932720000 & 0.366675000 \\ \mathrm{~N} & -0.144548000 & 3.928184000 & -0.644949000 \\ \mathrm{C} & -1.757412000 & -0.934879000 & -0.346943000 \\ \mathrm{C} & -1.949092000 & -1.002354000 & 1.038056000 \\ \mathrm{C} & -3.214117000 & -0.787245000 & 1.586599000\end{array}$




$\begin{array}{lrrr}\mathrm{C} & -4.301767000 & -0.503223000 & 0.758397000 \\ \mathrm{C} & -4.118574000 & -0.432691000 & -0.623458000 \\ \mathrm{C} & -2.852081000 & -0.643629000 & -1.171616000 \\ \mathrm{C} & 2.707476000 & -2.376715000 & -0.599783000 \\ \mathrm{O} & 3.346521000 & -3.469924000 & 0.030488000 \\ \mathrm{H} & 2.266723000 & 4.093261000 & 0.634533000 \\ \mathrm{H} & 3.588815000 & 2.096679000 & 1.376858000 \\ \mathrm{H} & -0.692458000 & 1.320977000 & -0.903964000 \\ \mathrm{H} & -0.342871000 & -0.724563000 & -1.960456000 \\ \mathrm{H} & -0.312380000 & -2.291625000 & -1.187668000 \\ \mathrm{H} & 2.064934000 & -1.597271000 & 1.296837000 \\ \mathrm{H} & 0.962023000 & -2.786373000 & 0.590163000 \\ \mathrm{H} & -1.056404000 & 3.589974000 & -0.972036000 \\ \mathrm{H} & -0.312795000 & 4.587608000 & 0.124074000 \\ \mathrm{H} & -1.109301000 & -1.224303000 & 1.689915000 \\ \mathrm{H} & -3.351091000 & -0.848420000 & 2.661936000 \\ \mathrm{H} & -5.286059000 & -0.342621000 & 1.186951000 \\ \mathrm{H} & -4.960110000 & -0.218119000 & -1.275239000 \\ \mathrm{H} & -2.718134000 & -0.599219000 & -2.250599000 \\ \mathrm{H} & 2.263470000 & -2.660419000 & -1.568522000 \\ \mathrm{H} & 3.393208000 & -1.535468000 & -0.777420000 \\ \mathrm{H} & 4.090064000 & -3.755328000 & -0.514851000 \\ \mathrm{H} & 0.291887000 & 4.452817000 & -1.412773000\end{array}$

\section{Rotamer XV}

11

$\begin{array}{llll}\text { C } & 1.907973000 & 3.088339000 & 0.447630000\end{array}$

$\begin{array}{llll}\mathrm{C} & 2.640170000 & 1.965797000 & 0.854903000\end{array}$

$\begin{array}{llll}\mathrm{N} & 2.267330000 & 0.709200000 & 0.650116000\end{array}$

$\begin{array}{llll}\text { C } & 1.104765000 & 0.446526000 & 0.009403000\end{array}$

$\begin{array}{llll}\text { C } & 0.266428000 & 1.512192000 & -0.444029000\end{array}$

$\begin{array}{llll}\text { C } & 0.714891000 & 2.789661000 & -0.197138000\end{array}$

$\begin{array}{llll}\mathrm{N} & 0.765018000 & -0.855131000 & -0.172560000\end{array}$

$\begin{array}{llll}\text { C } & -0.400327000 & -1.223030000 & -0.981681000\end{array}$

$\begin{array}{llll}\text { C } & 1.611113000 & -1.930302000 & 0.367211000\end{array}$

$\begin{array}{llll}\mathrm{N} & -0.152278000 & 3.926744000 & -0.646193000\end{array}$

C $\quad-1.755613000 \quad-0.937821000 \quad-0.346317000$

$\begin{array}{llll}\text { C } & -1.947151000 & -1.002994000 & 1.038806000\end{array}$

$\begin{array}{llll}\text { C } & -3.212516000 & -0.788864000 & 1.586962000\end{array}$

$\begin{array}{llll}\text { C } & -4.300628000 & -0.508083000 & 0.758274000\end{array}$

$\begin{array}{llll}\text { C } & -4.117565000 & -0.439814000 & -0.623717000\end{array}$

$\begin{array}{llll}\text { C } & -2.850772000 & -0.649810000 & -1.171503000\end{array}$

$\begin{array}{llll}\text { C } & 2.712194000 & -2.372377000 & -0.599376000\end{array}$

$\begin{array}{llll}\text { O } & 3.353199000 & -3.464497000 & 0.030783000\end{array}$

$\mathrm{H} \quad 2.258180000 \quad 4.097050000 \quad 0.634196000$

$\begin{array}{llll}\mathrm{H} & 3.584305000 & 2.103295000 & 1.376924000\end{array}$

$\mathrm{H} \quad-0.694466000 \quad 1.318585000 \quad-0.905491000$

$\mathrm{H} \quad-0.341511000 \quad-0.727929000 \quad-1.960221000$

$\mathrm{H} \quad-0.308944000 \quad-2.293884000 \quad-1.185278000$

$\begin{array}{llll}\mathrm{H} & 2.068438000 & -1.593673000 & 1.297201000\end{array}$ 


$\begin{array}{rrrr}\mathrm{H} & 0.967845000 & -2.785212000 & 0.591014000 \\ \mathrm{H} & -1.061986000 & 3.586232000 & -0.976843000 \\ \mathrm{H} & -0.325099000 & 4.584054000 & 0.123635000 \\ \mathrm{H} & -1.107037000 & -1.222461000 & 1.691079000 \\ \mathrm{H} & -3.349374000 & -0.848294000 & 2.662413000 \\ \mathrm{H} & -5.285172000 & -0.348289000 & 1.186550000 \\ \mathrm{H} & -4.959451000 & -0.227806000 & -1.275884000 \\ \mathrm{H} & -2.716935000 & -0.607229000 & -2.250573000 \\ \mathrm{H} & 2.268513000 & -2.656799000 & -1.568050000 \\ \mathrm{H} & 3.396481000 & -1.529971000 & -0.777100000 \\ \mathrm{H} & 4.097487000 & -3.748264000 & -0.514393000 \\ \mathrm{H} & 0.284863000 & 4.454361000 & -1.411559000\end{array}$

\section{Rotamer XVI}

11

C $\quad 1.909579000$

C 2.641072000

3.087432000

0.448082000

$\mathrm{N} \quad 2.267477000$

1.964547000

0.855670000

C $\quad 1.104780000$

0.708144000

0.651141000

C $\quad 0.267077000$

0.446029000

0.010442000

C $\quad 0.716338000$

1.512082000

$-0.443241000$

$\mathrm{N} \quad 0.764345000$

2.789336000

$-0.196671000$

C $\quad-0.400795000$

$-0.855475000$

$-0.171421000$

C $\quad 1.610635000$

$-1.222845000$

$-0.981090000$

$\mathrm{N} \quad-0.149875000$

$-1.931044000$

0.367329000

C $\quad-1.756233000$

3.926866000

$-0.646435000$

C $\quad-1.948711000$

$-0.937240000$

$-0.346244000$

C $\quad-3.214202000$

$-1.004532000$

1.038653000

C $\quad-4.301480000$

$-0.790027000$

1.586356000

$-0.506746000$

0.757403000

C $\quad-4.117465000$

$-0.436339000$

$-0.624345000$

C $\quad-2.850517000$

$-0.646694000$

$-1.171668000$

C 2.711137000

$-2.372580000$

$-0.600147000$

O 3.352478000

$-3.465127000$

0.028922000

$\mathrm{H} \quad 2.260420000$

4.095971000

0.634389000

$\mathrm{H} \quad 3.585275000$

2.101587000

1.377686000

$\mathrm{H} \quad-0.693799000$

1.318842000

$-0.904855000$

$\mathrm{H} \quad-0.341440000$

$-0.727672000$

$-1.959558000$

H $\quad-0.309729000$

$-2.293719000$

$-1.184704000$

$\mathrm{H} \quad 2.068468000$

$-1.594944000$

1.297260000

$\mathrm{H} \quad 0.967426000$

$-2.786026000$

0.591030000

$\mathrm{H}-1.059889000$

3.586936000

$-0.976848000$

H $\quad-0.322094000$

4.584871000

0.122934000

H $\quad-1.109142000$

$-1.225851000$

1.691021000

$\mathrm{H} \quad-3.351828000$

$-0.851091000$

2.661615000

$\mathrm{H} \quad-5.286136000$

$-0.346642000$

1.185309000

$\mathrm{H} \quad-4.958719000$

$-0.222347000$

$-1.276679000$

H $\quad-2.715908000$

$-0.602462000$

$-2.250576000$

$\mathrm{H} \quad 2.266899000$

$-2.656369000$

$-1.568761000$

H 3.395345000

$-1.530074000$

$-0.777727000$

H 4.096408000

$-3.748587000$

$-0.516903000$ 


\section{Adduct XVIIa}

$\begin{array}{lrrr}11 & & & \\ \mathrm{C} & 3.62119 & -1.04846 & -0.25570 \\ \mathrm{C} & 2.59388 & -1.04100 & -1.15785 \\ \mathrm{~N} & 1.52358 & -0.14214 & -1.05997 \\ \mathrm{C} & 1.46753 & 0.70186 & 0.07051 \\ \mathrm{C} & 2.46648 & 0.67633 & 1.03063 \\ \mathrm{C} & 3.57565 & -0.17916 & 0.87427 \\ \mathrm{~N} & 0.40299 & 1.63704 & 0.21826 \\ \mathrm{C} & -0.72918 & 1.20611 & 1.04729 \\ \mathrm{C} & 0.03716 & 2.32748 & -1.03963 \\ \mathrm{~N} & 4.56830 & -0.25016 & 1.86156 \\ \mathrm{C} & -2.15049 & 1.40436 & 0.57869 \\ \mathrm{C} & -2.50998 & 1.80827 & -0.70511 \\ \mathrm{C} & -3.85404 & 1.96536 & -1.04537 \\ \mathrm{C} & -4.85008 & 1.71235 & -0.10700 \\ \mathrm{C} & -4.49738 & 1.30344 & 1.17921 \\ \mathrm{C} & -3.15778 & 1.15132 & 1.51980 \\ \mathrm{C} & 1.07995 & 3.42504 & -1.33392 \\ \mathrm{O} & 2.07110 & 2.94421 & -2.21552 \\ \mathrm{H} & 4.46323 & -1.72174 & -0.37862 \\ \mathrm{H} & 2.55809 & -1.70089 & -2.02537 \\ \mathrm{H} & 2.38343 & 1.31501 & 1.90759 \\ \mathrm{H} & -0.57885 & 0.12245 & 1.28961 \\ \mathrm{H} & -0.60002 & 1.74995 & 2.02249 \\ \mathrm{H} & -0.01094 & 1.63910 & -1.91355 \\ \mathrm{H} & -0.96995 & 2.78141 & -0.92844 \\ \mathrm{H} & 4.65005 & 0.54556 & 2.46419 \\ \mathrm{H} & 5.45637 & -0.60773 & 1.56817 \\ \mathrm{H} & -1.75117 & 2.00033 & -1.46606 \\ \mathrm{H} & -4.12023 & 2.28869 & -2.05158 \\ \mathrm{H} & -5.89734 & 1.83998 & -0.37132 \\ \mathrm{H} & -5.27214 & 1.11146 & 1.91966 \\ \mathrm{H} & -2.89183 & 0.84083 & 2.52893 \\ \mathrm{H} & 0.63615 & 4.26750 & -1.89420 \\ \mathrm{H} & 1.56119 & 3.79870 & -0.41251 \\ \mathrm{H} & 2.47437 & 2.12268 & -1.85951 \\ \mathrm{O} & -0.70708 & -1.67477 & -2.35714 \\ \mathrm{H} & 0.65615 & -0.41249 & -1.52023 \\ \mathrm{H} & -1.45831 & -1.18656 & -2.70559 \\ & -1.06691 & -2.45895 & -1.94947 \\ & & & \end{array}$

\section{Adduct XVIIb}

$\begin{array}{llll}11 & & & \\ \text { C } & 3.64432 & -0.79763 & -0.46307 \\ \text { C } & 2.55138 & -0.92460 & -1.30824 \\ \text { N } & 1.31291 & -0.28020 & -1.03591 \\ \text { C } & 1.27770 & 0.60235 & 0.07489\end{array}$




$\begin{array}{lrrr}\mathrm{C} & 2.39667 & 0.77025 & 0.87917 \\ \mathrm{C} & 3.62232 & 0.11003 & 0.61715 \\ \mathrm{~N} & 0.13646 & 1.43901 & 0.28309 \\ \mathrm{C} & -1.08310 & 0.67679 & 0.71728 \\ \mathrm{C} & 0.16366 & 2.62745 & -0.60072 \\ \mathrm{~N} & 4.70005 & 0.24616 & 1.46577 \\ \mathrm{C} & -2.43048 & 1.28043 & 0.40109 \\ \mathrm{C} & -3.45279 & 0.58534 & -0.29755 \\ \mathrm{C} & -4.68414 & 1.18268 & -0.52118 \\ \mathrm{C} & -5.04736 & 2.37657 & 0.15738 \\ \mathrm{C} & -4.06347 & 3.00718 & 0.93804 \\ \mathrm{C} & -2.71845 & 2.51676 & 0.98111 \\ \mathrm{C} & 1.53930 & 3.36070 & -0.38241 \\ \mathrm{O} & 2.44785 & 2.88854 & -1.33148 \\ \mathrm{H} & 4.53348 & -1.40324 & -0.70462 \\ \mathrm{H} & 2.59598 & -1.52882 & -2.27155 \\ \mathrm{H} & 2.27006 & 1.50940 & 1.66653 \\ \mathrm{H} & -1.03523 & -0.40325 & 0.37327 \\ \mathrm{H} & -1.04517 & 0.68274 & 1.85458 \\ \mathrm{H} & 0.18816 & 2.39493 & -1.62162 \\ \mathrm{H} & -0.63352 & 3.29863 & -0.29261 \\ \mathrm{H} & 4.76017 & 1.08487 & 2.00944 \\ \mathrm{H} & 5.58617 & 0.01839 & 1.03225 \\ \mathrm{H} & -3.19508 & -0.37312 & -0.75994 \\ \mathrm{H} & -5.32745 & 0.70382 & -1.26870 \\ \mathrm{H} & -6.05808 & 2.73622 & -0.04856 \\ \mathrm{H} & -4.26898 & 3.97116 & 1.46890 \\ \mathrm{H} & -1.90771 & 3.18363 & 1.24902 \\ \mathrm{H} & 1.38249 & 4.42576 & -0.58440 \\ \mathrm{H} & 1.85641 & 3.19725 & 0.65278 \\ \mathrm{H} & 2.77319 & 1.98519 & -1.09619 \\ \mathrm{O} & 0.02107 & -3.26274 & -3.36529 \\ \mathrm{H} & 0.60463 & -0.22241 & -1.72889 \\ \mathrm{H} & 0.24015 & -2.49315 & -2.83686 \\ \mathrm{H} & 0.57734 & -3.94747 & -3.03831\end{array}$

\section{Adduct XVIIIa}

$\begin{array}{lrrr}11 & & & \\ \mathrm{C} & 0.99277 & -3.24308 & -1.94808 \\ \mathrm{C} & 0.71184 & -3.37751 & -0.53601 \\ \mathrm{~N} & 0.03027 & -2.53718 & 0.17135 \\ \mathrm{C} & -0.56848 & -1.37310 & -0.47315 \\ \mathrm{C} & 0.19619 & -0.89285 & -1.75421 \\ \mathrm{C} & 0.71982 & -2.05016 & -2.55001 \\ \mathrm{~N} & -0.56446 & -0.18638 & 0.42797 \\ \mathrm{C} & -1.32181 & -0.30287 & 1.68762 \\ \mathrm{C} & -0.86519 & 1.05343 & -0.31942 \\ \mathrm{~N} & 0.93783 & -1.82720 & -3.88628 \\ \mathrm{C} & -2.41117 & 0.71822 & 1.90787 \\ \mathrm{C} & -3.70663 & 0.47762 & 1.44817\end{array}$




$\begin{array}{rrrr}\mathrm{C} & -4.70783 & 1.42575 & 1.64406 \\ \mathrm{C} & -4.42059 & 2.61805 & 2.30686 \\ \mathrm{C} & -3.13202 & 2.85429 & 2.78260 \\ \mathrm{C} & -2.13073 & 1.90508 & 2.58855 \\ \mathrm{C} & -1.13344 & 2.17534 & 0.70601 \\ \mathrm{O} & -1.12106 & 3.42661 & 0.05218 \\ \mathrm{H} & 1.40872 & -4.08877 & -2.47098 \\ \mathrm{H} & 1.10150 & -4.26662 & -0.01659 \\ \mathrm{H} & -0.46529 & -0.23150 & -2.34259 \\ \mathrm{H} & -1.74850 & -1.33656 & 1.74388 \\ \mathrm{H} & -0.56975 & -0.25073 & 2.51163 \\ \mathrm{H} & 0.00481 & 1.35218 & -0.94628 \\ \mathrm{H} & -1.72212 & 0.95529 & -1.02123 \\ \mathrm{H} & 0.78193 & -0.93536 & -4.29929 \\ \mathrm{H} & 1.33261 & -2.53565 & -4.46529 \\ \mathrm{H} & -3.93147 & -0.46213 & 0.93297 \\ \mathrm{H} & -5.71702 & 1.22994 & 1.28564 \\ \mathrm{H} & -5.20273 & 3.35786 & 2.46252 \\ \mathrm{H} & -2.90863 & 3.77891 & 3.31120 \\ \mathrm{H} & -1.13003 & 2.08940 & 2.97446 \\ \mathrm{H} & -2.09376 & 2.04735 & 1.23073 \\ \mathrm{H} & -0.31504 & 2.26311 & 1.44440 \\ \mathrm{H} & -1.76688 & 3.44408 & -0.68022 \\ \mathrm{H} & 1.05046 & -0.24990 & -1.43264 \\ \mathrm{O} & -3.88127 & -1.68371 & 0.53428 \\ \mathrm{H} & -1.61923 & -1.67086 & -0.77029 \\ \mathrm{H} & -2.92062 & -1.70852 & 0.55293 \\ \mathrm{H} & -4.14553 & -1.90057 & 1.43101\end{array}$

\section{Adduct XVIIIb}

$\begin{array}{lrrr}11 & & & \\ \mathrm{C} & -1.60477 & -2.33444 & -0.55359 \\ \mathrm{C} & -2.57454 & -1.25009 & -0.73339 \\ \mathrm{~N} & -2.32931 & -0.00951 & -0.99614 \\ \mathrm{C} & -0.94756 & 0.43944 & -1.18363 \\ \mathrm{C} & 0.01426 & -0.72227 & -1.43468 \\ \mathrm{C} & -0.30009 & -1.97050 & -0.65754 \\ \mathrm{~N} & -0.35742 & 1.32047 & -0.11181 \\ \mathrm{C} & -0.47424 & 0.93846 & 1.33523 \\ \mathrm{C} & -0.95063 & 2.60947 & -0.27515 \\ \mathrm{~N} & 0.69836 & -2.74446 & -0.04253 \\ \mathrm{C} & -1.54729 & 1.49880 & 2.17835 \\ \mathrm{C} & -2.03869 & 2.81488 & 2.43332 \\ \mathrm{C} & -3.30936 & 3.02613 & 3.00593 \\ \mathrm{C} & -4.08837 & 1.90971 & 3.38859 \\ \mathrm{C} & -3.37508 & 0.69350 & 3.46606 \\ \mathrm{C} & -2.17281 & 0.44031 & 2.80533 \\ \mathrm{C} & -0.91203 & 3.40132 & -1.62222 \\ \mathrm{O} & -0.27816 & 2.70189 & -2.59939 \\ \mathrm{H} & -1.90660 & -3.32638 & -0.21548\end{array}$




$\begin{array}{cccc}\mathrm{H} & -3.62163 & -1.46221 & -0.45489 \\ \mathrm{H} & 0.12095 & -0.96516 & -2.49315 \\ \mathrm{H} & -0.59995 & -0.18041 & 1.51217 \\ \mathrm{H} & 0.57921 & 1.22362 & 1.63349 \\ \mathrm{H} & -2.05146 & 2.61881 & 0.02032 \\ \mathrm{H} & -0.25569 & 3.28306 & 0.09531 \\ \mathrm{H} & 1.65073 & -2.51934 & -0.22408 \\ \mathrm{H} & 0.52471 & -3.54187 & 0.52323 \\ \mathrm{H} & -1.38510 & 3.62746 & 2.01927 \\ \mathrm{H} & -3.77358 & 3.95171 & 3.05655 \\ \mathrm{H} & -5.09450 & 2.05365 & 3.62993 \\ \mathrm{H} & -3.85536 & -0.17777 & 3.80395 \\ \mathrm{H} & -1.82744 & -0.62801 & 2.67934 \\ \mathrm{H} & -2.02728 & 3.45557 & -1.80250 \\ \mathrm{H} & -0.35341 & 4.32123 & -1.55189 \\ \mathrm{H} & 0.64146 & 2.60270 & -2.35329 \\ \mathrm{H} & 1.05480 & -0.36124 & -1.18470 \\ \mathrm{O} & -1.04365 & -10.36667 & -2.18387 \\ \mathrm{H} & -1.02948 & 1.04718 & -2.15179 \\ \mathrm{H} & -0.99773 & -10.35549 & -3.10468 \\ \mathrm{H} & -1.66544 & -11.05041 & -2.01732\end{array}$

\section{Adduct XIXa}

$\begin{array}{lrrr}11 & & & \\ \mathrm{C} & 3.16094 & -1.66078 & -0.38964 \\ \mathrm{C} & 2.75238 & -1.67435 & 0.94082 \\ \mathrm{~N} & 1.76443 & -0.90913 & 1.43319 \\ \mathrm{C} & 1.12647 & -0.04388 & 0.57997 \\ \mathrm{C} & 1.46286 & 0.04145 & -0.78362 \\ \mathrm{C} & 2.49194 & -0.78172 & -1.27213 \\ \mathrm{~N} & 0.19923 & 0.81793 & 1.15946 \\ \mathrm{C} & -0.94628 & 0.25934 & 1.89845 \\ \mathrm{C} & -0.05688 & 2.11123 & 0.50629 \\ \mathrm{~N} & 2.82855 & -0.74255 & -2.60386 \\ \mathrm{C} & -2.25523 & 0.91237 & 1.53356 \\ \mathrm{C} & -2.84480 & 0.64527 & 0.29721 \\ \mathrm{C} & -4.04279 & 1.26283 & -0.05129 \\ \mathrm{C} & -4.65995 & 2.14173 & 0.83809 \\ \mathrm{C} & -4.07802 & 2.39937 & 2.07780 \\ \mathrm{C} & -2.87625 & 1.78589 & 2.42689 \\ \mathrm{C} & -0.09099 & 3.22492 & 1.57416 \\ \mathrm{O} & 0.88775 & 4.16060 & 1.14190 \\ \mathrm{H} & 3.22734 & -2.33841 & 1.67559 \\ \mathrm{H} & 0.95189 & 0.73076 & -1.44611 \\ \mathrm{H} & -1.00391 & -0.84576 & 1.70788 \\ \mathrm{H} & -0.72383 & 0.36659 & 2.98560 \\ \mathrm{H} & 0.72333 & 2.35554 & -0.25747 \\ \mathrm{H} & -1.01631 & 2.07854 & -0.05359 \\ \mathrm{H} & 2.39710 & -0.08399 & -3.21246 \\ \mathrm{H} & 3.60318 & -1.27105 & -2.95445\end{array}$




$\begin{array}{lrrr}\mathrm{H} & -2.36021 & -0.04936 & -0.39394 \\ \mathrm{H} & -4.49747 & 1.05783 & -1.01839 \\ \mathrm{H} & -5.59524 & 2.62447 & 0.56522 \\ \mathrm{H} & -4.56174 & 3.08039 & 2.77393 \\ \mathrm{H} & -2.42928 & 1.98890 & 3.39707 \\ \mathrm{H} & -1.06693 & 3.73601 & 1.60913 \\ \mathrm{H} & 0.17381 & 2.84445 & 2.57699 \\ \mathrm{H} & 1.04395 & 4.83478 & 1.82777 \\ \mathrm{O} & 5.18791 & -2.38801 & -3.48890 \\ \mathrm{H} & 4.98992 & -3.05154 & -4.27439 \\ \mathrm{H} & 6.05255 & -1.83732 & -3.70458 \\ \mathrm{H} & 3.95534 & -2.30184 & -0.73661 \\ \mathrm{H} & 5.33577 & -2.93259 & -2.60593\end{array}$

\section{Adduct XIXb}

$\begin{array}{lrrr}11 & & & \\ \mathrm{C} & 1.78442 & -0.43386 & -1.82915 \\ \mathrm{C} & 0.99985 & 0.59330 & -2.34590 \\ \mathrm{~N} & 0.43177 & 1.56450 & -1.60525 \\ \mathrm{C} & 0.55776 & 1.46786 & -0.24441 \\ \mathrm{C} & 1.34270 & 0.47209 & 0.37701 \\ \mathrm{C} & 1.96794 & -0.48822 & -0.42640 \\ \mathrm{~N} & -0.08768 & 2.42180 & 0.53594 \\ \mathrm{C} & -0.52515 & 2.01835 & 1.88632 \\ \mathrm{C} & -0.79195 & 3.56418 & -0.07929 \\ \mathrm{~N} & 2.73696 & -1.47999 & 0.13085 \\ \mathrm{C} & -1.42086 & 0.79682 & 1.90451 \\ \mathrm{C} & -2.45333 & 0.69959 & 0.96902 \\ \mathrm{C} & -3.21318 & -0.46373 & 0.89577 \\ \mathrm{C} & -2.91091 & -1.54340 & 1.72353 \\ \mathrm{C} & -1.91544 & -1.43326 & 2.69382 \\ \mathrm{C} & -1.18489 & -0.24965 & 2.80162 \\ \mathrm{C} & 0.04550 & 4.85809 & 0.10184 \\ \mathrm{O} & 0.63579 & 5.20727 & -1.13933 \\ \mathrm{H} & 0.79936 & 0.66965 & -3.42641 \\ \mathrm{H} & 1.45112 & 0.44166 & 1.47671 \\ \mathrm{H} & 0.39291 & 1.84904 & 2.50253 \\ \mathrm{H} & -1.04106 & 2.87080 & 2.36158 \\ \mathrm{H} & -0.94171 & 3.39707 & -1.18101 \\ \mathrm{H} & -1.81203 & 3.67905 & 0.33423 \\ \mathrm{H} & 2.83861 & -1.55191 & 1.11622 \\ \mathrm{H} & 3.15305 & -2.19306 & -0.42205 \\ \mathrm{H} & -2.62823 & 1.49982 & 0.26503 \\ \mathrm{H} & -4.01414 & -0.54705 & 0.16030 \\ \mathrm{H} & -3.45190 & -2.48110 & 1.60235 \\ \mathrm{H} & -1.69540 & -2.27150 & 3.35264 \\ \mathrm{H} & -0.40263 & -0.16044 & 3.55429 \\ \mathrm{H} & -0.59435 & 5.72064 & 0.34802 \\ \mathrm{H} & 0.82899 & 4.75705 & 0.85880 \\ \mathrm{H} & 1.16222 & 4.44955 & -1.48481\end{array}$




$\begin{array}{llll}\mathrm{O} & 4.48777 & -5.24991 & 0.24591 \\ \mathrm{H} & 4.02913 & -6.17584 & 0.39169 \\ \mathrm{H} & 4.73241 & -5.14693 & -0.76646 \\ \mathrm{H} & 2.22535 & -1.17192 & -2.47467 \\ \mathrm{H} & 5.35652 & -5.20313 & 0.82590\end{array}$

\section{Adduct XX}

01

C $\quad 16.771969000 \quad-24.511120000 \quad-1.331926000$

$\begin{array}{llll}\text { C } & 16.538732000 & -25.779875000 & -1.780298000\end{array}$

$\begin{array}{llll}\mathrm{N} & 17.458988000 & -26.766956000 & -1.693007000\end{array}$

C $\quad 18.693241000 \quad-26.559921000 \quad-1.150496000$

$\begin{array}{llll}\text { C } & 18.990715000 & -25.259178000 & -0.685989000\end{array}$

$\begin{array}{llll}\text { C } & 18.043864000 & -24.234441000 & -0.761614000\end{array}$

$\begin{array}{llll}\mathrm{N} & 19.580874000 & -27.588602000 & -1.082270000\end{array}$

C $\quad 19.314552000 \quad-28.964049000 \quad-1.582462000$

$\begin{array}{llll}\text { C } & 20.892123000 & -27.386804000 & -0.469055000\end{array}$

N $\quad 18.316603000 \quad-22.988455000 \quad-0.274420000$

C $\quad 19.074480000 \quad-29.966488000 \quad-0.468632000$

$\begin{array}{llll}\text { C } & 17.794152000 & -30.116284000 & 0.083767000\end{array}$

$\begin{array}{llll}\text { C } & 17.579733000 & -31.020318000 & 1.123336000\end{array}$

$\begin{array}{llll}\text { C } & 18.634553000 & -31.789310000 & 1.620715000\end{array}$

$\begin{array}{llll}\text { C } & 19.906135000 & -31.663788000 & 1.061859000\end{array}$

$\begin{array}{llll}\text { C } & 20.120858000 & -30.759737000 & 0.019824000\end{array}$

$\begin{array}{llll}\text { C } & 21.931042000 & -26.845053000 & -1.454479000\end{array}$

O $\quad 23.129465000 \quad-26.665358000 \quad-0.714720000$

$\mathrm{H} \quad 16.009195000 \quad-23.745827000 \quad-1.411138000$

$\mathrm{H} \quad 15.609061000 \quad-26.101790000 \quad-2.238348000$

$\mathrm{H} \quad 19.962948000 \quad-25.041247000 \quad-0.267440000$

H $\quad 18.464963000 \quad-28.957370000 \quad-2.268777000$

$\mathrm{H} \quad 20.194128000 \quad-29.258861000 \quad-2.165374000$

H $\quad 20.820695000 \quad-26.720806000 \quad 0.394765000$

H $\quad 21.233689000 \quad-28.346363000 \quad-0.078024000$

$\begin{array}{llll}\mathrm{H} & 19.262266000 & -22.745395000 & -0.026647000\end{array}$

$\mathrm{H} \quad 17.707960000 \quad-22.222282000 \quad-0.512583000$

H $\quad 16.961673000 \quad-29.560983000 \quad-0.338829000$

$\begin{array}{llll}\mathrm{H} & 16.581510000 & -31.135756000 & 1.535431000\end{array}$

$\mathrm{H} \quad 18.461809000 \quad-32.494978000 \quad 2.428281000$

H $\quad 20.727592000 \quad-32.272937000 \quad 1.427920000$

H $\quad 21.108605000 \quad-30.686367000 \quad-0.430472000$

$\mathrm{H} \quad 22.059364000 \quad-27.561579000 \quad-2.279939000$

$\mathrm{H} \quad 21.575916000 \quad-25.900686000 \quad-1.895116000$

$\mathrm{H} \quad 23.834623000 \quad-26.428008000 \quad-1.329376000$

$\mathrm{H} \quad 17.069581000 \quad-27.704065000 \quad-2.118839000$

Cl $\quad 16.001884000 \quad-28.932057000 \quad-2.929200000$

\section{Adduct XXI}

01

$\begin{array}{llll}\text { C } & 3.459923000 & -1.930862000 & 0.192286000\end{array}$

$\begin{array}{llll}\text { C } & 2.359921000 & -2.251496000 & 0.935498000\end{array}$ 


$\begin{array}{lrrr} & & & \\ \mathrm{N} & 1.274178000 & -1.449523000 & 1.018120000 \\ \mathrm{C} & 1.204831000 & -0.255299000 & 0.362706000 \\ \mathrm{C} & 2.324464000 & 0.130803000 & -0.407717000 \\ \mathrm{C} & 3.447283000 & -0.694677000 & -0.508587000 \\ \mathrm{~N} & 0.086985000 & 0.511764000 & 0.475568000 \\ \mathrm{C} & -1.101897000 & 0.150866000 & 1.293660000 \\ \mathrm{C} & -0.011575000 & 1.776305000 & -0.250667000 \\ \mathrm{~N} & 4.511810000 & -0.340854000 & -1.286926000 \\ \mathrm{C} & -2.287678000 & -0.293005000 & 0.456925000 \\ \mathrm{C} & -2.398908000 & -1.627579000 & 0.040505000 \\ \mathrm{C} & -3.476026000 & -2.029125000 & -0.748508000 \\ \mathrm{C} & -4.457139000 & -1.109559000 & -1.126897000 \\ \mathrm{C} & -4.367037000 & 0.214413000 & -0.697949000 \\ \mathrm{C} & -3.289433000 & 0.616485000 & 0.093471000 \\ \mathrm{C} & 0.605003000 & 2.949809000 & 0.515212000 \\ \mathrm{O} & 0.470654000 & 4.089103000 & -0.321274000 \\ \mathrm{H} & 4.310254000 & -2.600372000 & 0.142667000 \\ \mathrm{H} & 2.262503000 & -3.163572000 & 1.515457000 \\ \mathrm{H} & 2.324839000 & 1.077819000 & -0.928344000 \\ \mathrm{H} & -0.841065000 & -0.626995000 & 2.014590000 \\ \mathrm{H} & -1.366203000 & 1.044712000 & 1.869445000 \\ \mathrm{H} & 0.450631000 & 1.696649000 & -1.238007000 \\ \mathrm{H} & -1.067849000 & 1.980638000 & -0.431856000 \\ \mathrm{H} & 4.577652000 & 0.598165000 & -1.645611000 \\ \mathrm{H} & 5.379551000 & -0.843174000 & -1.192657000 \\ \mathrm{H} & -1.667976000 & -2.355874000 & 0.380071000 \\ \mathrm{H} & -3.557322000 & -3.067773000 & -1.055431000 \\ \mathrm{H} & -5.296388000 & -1.427931000 & -1.738674000 \\ \mathrm{H} & -5.136450000 & 0.931433000 & -0.969503000 \\ \mathrm{H} & -3.239428000 & 1.643900000 & 0.448018000 \\ \mathrm{H} & 0.078457000 & 3.077083000 & 1.473238000 \\ \mathrm{H} & 1.659197000 & 2.734970000 & 0.748928000 \\ \mathrm{H} & 0.751098000 & 4.867285000 & 0.175887000 \\ \mathrm{H} & 0.509217000 & -1.892553000 & 1.673678000 \\ \mathrm{Cl} & -0.369998000 & -3.001947000 & 2.814191000\end{array}$

\section{Adduct XXII}

01

$\begin{array}{lrrr}\mathrm{C} & 3.460773000 & -1.929249000 & 0.192552000 \\ \mathrm{C} & 2.360674000 & -2.250765000 & 0.935237000 \\ \mathrm{~N} & 1.274521000 & -1.449349000 & 1.017879000 \\ \mathrm{C} & 1.204825000 & -0.254843000 & 0.363018000 \\ \mathrm{C} & 2.324534000 & 0.132163000 & -0.406842000 \\ \mathrm{C} & 3.447780000 & -0.692734000 & -0.507732000 \\ \mathrm{~N} & 0.086577000 & 0.511634000 & 0.475873000 \\ \mathrm{C} & -1.102418000 & 0.149761000 & 1.293370000 \\ \mathrm{C} & -0.012344000 & 1.776476000 & -0.249789000 \\ \mathrm{~N} & 4.512399000 & -0.338030000 & -1.285545000 \\ \mathrm{C} & -2.287687000 & -0.294281000 & 0.456000000 \\ \mathrm{C} & -2.398104000 & -1.628703000 & 0.038874000\end{array}$




$\begin{array}{lrrr}\mathrm{C} & -3.474746000 & -2.030392000 & -0.750715000 \\ \mathrm{C} & -4.456186000 & -1.111126000 & -1.128988000 \\ \mathrm{C} & -4.366894000 & 0.212676000 & -0.699348000 \\ \mathrm{C} & -3.289767000 & 0.614890000 & 0.092650000 \\ \mathrm{C} & 0.603408000 & 2.949908000 & 0.516864000 \\ \mathrm{O} & 0.468782000 & 4.089542000 & -0.319113000 \\ \mathrm{H} & 4.311440000 & -2.598330000 & 0.142897000 \\ \mathrm{H} & 2.263495000 & -3.163167000 & 1.514724000 \\ \mathrm{H} & 2.324632000 & 1.079429000 & -0.927015000 \\ \mathrm{H} & -0.841462000 & -0.628323000 & 2.014015000 \\ \mathrm{H} & -1.367362000 & 1.043199000 & 1.869496000 \\ \mathrm{H} & 0.450236000 & 1.697517000 & -1.237009000 \\ \mathrm{H} & -1.068654000 & 1.980388000 & -0.431239000 \\ \mathrm{H} & 4.577915000 & 0.601194000 & -1.643751000 \\ \mathrm{H} & 5.380348000 & -0.839980000 & -1.191223000 \\ \mathrm{H} & -1.666927000 & -2.356801000 & 0.378331000 \\ \mathrm{H} & -3.555416000 & -3.068926000 & -1.058185000 \\ \mathrm{H} & -5.295063000 & -1.429611000 & -1.741217000 \\ \mathrm{H} & -5.136569000 & 0.929448000 & -0.970814000 \\ \mathrm{H} & -3.240398000 & 1.642154000 & 0.447726000 \\ \mathrm{H} & 0.076481000 & 3.076460000 & 1.474777000 \\ \mathrm{H} & 1.657629000 & 2.735469000 & 0.750828000 \\ \mathrm{H} & 0.748687000 & 4.867619000 & 0.178517000 \\ \mathrm{H} & 0.509548000 & -1.893064000 & 1.672961000 \\ \mathrm{Cl} & -0.369526000 & -3.003435000 & 2.812630000\end{array}$

\section{Adduct XXIV}

01

$\begin{array}{lrrr}\mathrm{C} & 3.459860000 & -1.930941000 & 0.192585000 \\ \mathrm{C} & 2.359668000 & -2.251785000 & 0.935423000 \\ \mathrm{~N} & 1.273911000 & -1.449827000 & 1.018008000 \\ \mathrm{C} & 1.204741000 & -0.255408000 & 0.362932000 \\ \mathrm{C} & 2.324572000 & 0.130912000 & -0.407095000 \\ \mathrm{C} & 3.447409000 & -0.694549000 & -0.507926000 \\ \mathrm{~N} & 0.086875000 & 0.511634000 & 0.475740000 \\ \mathrm{C} & -1.102219000 & 0.150502000 & 1.293421000 \\ \mathrm{C} & -0.011490000 & 1.776390000 & -0.250146000 \\ \mathrm{~N} & 4.512135000 & -0.340507000 & -1.285894000 \\ \mathrm{C} & -2.287793000 & -0.293099000 & 0.456250000 \\ \mathrm{C} & -2.398923000 & -1.627542000 & 0.039384000 \\ \mathrm{C} & -3.475847000 & -2.028837000 & -0.750023000 \\ \mathrm{C} & -4.456862000 & -1.109148000 & -1.128368000 \\ \mathrm{C} & -4.366862000 & 0.214689000 & -0.698983000 \\ \mathrm{C} & -3.289452000 & 0.616509000 & 0.092832000 \\ \mathrm{C} & 0.604906000 & 2.949662000 & 0.516235000 \\ \mathrm{O} & 0.470776000 & 4.089206000 & -0.319946000 \\ \mathrm{H} & 4.310198000 & -2.600445000 & 0.142982000 \\ \mathrm{H} & 2.262094000 & -3.164033000 & 1.515085000 \\ \mathrm{H} & 2.325087000 & 1.078082000 & -0.927442000 \\ \mathrm{H} & -0.841579000 & -0.627582000 & 2.014180000\end{array}$




$\begin{array}{lrrr}\mathrm{H} & -1.366660000 & 1.044176000 & 1.869412000 \\ \mathrm{H} & 0.450964000 & 1.697022000 & -1.237393000 \\ \mathrm{H} & -1.067716000 & 1.980787000 & -0.431541000 \\ \mathrm{H} & 4.578076000 & 0.598619000 & -1.644280000 \\ \mathrm{H} & 5.379848000 & -0.842860000 & -1.191551000 \\ \mathrm{H} & -1.668078000 & -2.355945000 & 0.378903000 \\ \mathrm{H} & -3.557070000 & -3.067387000 & -1.057292000 \\ \mathrm{H} & -5.295960000 & -1.427325000 & -1.740454000 \\ \mathrm{H} & -5.136203000 & 0.931798000 & -0.970504000 \\ \mathrm{H} & -3.239531000 & 1.643813000 & 0.447713000 \\ \mathrm{H} & 0.078121000 & 3.076656000 & 1.474168000 \\ \mathrm{H} & 1.659040000 & 2.734745000 & 0.750152000 \\ \mathrm{H} & 0.751100000 & 4.867238000 & 0.177516000 \\ \mathrm{H} & 0.508777000 & -1.893050000 & 1.673235000 \\ \mathrm{Cl} & -0.370745000 & -3.002781000 & 2.813180000\end{array}$

\section{Adduct XXV}

01

C $\quad 16.374740000 \quad-25.983255000 \quad-0.931234000$

$\begin{array}{llll}\text { C } & 16.817065000 & -26.955651000 & -1.914103000\end{array}$

$\begin{array}{llll}\mathrm{N} & 17.975207000 & -27.028148000 & -2.468202000\end{array}$

$\begin{array}{llll}\text { C } & 19.006562000 & -26.081899000 & -2.037330000\end{array}$

C $\quad 18.516308000 \quad-24.822051000 \quad-1.259493000$

$\begin{array}{llll}\text { C } & 17.181493000 & -24.948544000 & -0.585960000\end{array}$

$\begin{array}{llll}\mathrm{N} & 20.074127000 & -26.741699000 & -1.280936000\end{array}$

C $\quad 19.635764000 \quad-27.502218000 \quad-0.104956000$

$\begin{array}{llll}\text { C } & 21.087996000 & -27.405299000 & -2.106936000\end{array}$

$\begin{array}{llll}\mathrm{N} & 16.905745000 & -24.013587000 & 0.385434000\end{array}$

C $\quad 20.736270000 \quad-27.696287000 \quad 0.924515000$

$\begin{array}{llll}\text { C } & 21.613200000 & -26.654353000 & 1.254583000\end{array}$

$\begin{array}{llll}\text { C } & 22.580842000 & -26.824940000 & 2.243574000\end{array}$

C $\quad 22.685683000 \quad-28.041020000 \quad 2.922874000$

C $\quad 21.819803000 \quad-29.085729000 \quad 2.600171000$

$\begin{array}{llll}\text { C } & 20.856172000 & -28.913135000 & 1.604708000\end{array}$

C $\quad 20.645614000 \quad-28.627272000 \quad-2.922578000$

O $\quad 21.829002000 \quad-29.127765000 \quad-3.543416000$

$\mathrm{H} \quad 15.391045000 \quad-26.099170000 \quad-0.485810000$

$\mathrm{H} \quad 16.085906000 \quad-27.710100000 \quad-2.216660000$

$\mathrm{H} \quad 19.289595000 \quad-24.540151000 \quad-0.543935000$

$\mathrm{H} \quad 18.820562000 \quad-26.937411000 \quad 0.363152000$

$\mathrm{H} \quad 19.205364000 \quad-28.481982000 \quad-0.362010000$

$\mathrm{H} \quad 21.506740000 \quad-26.664977000 \quad-2.798271000$

$\begin{array}{llll}\mathrm{H} & 21.905014000 & -27.717892000 & -1.449560000\end{array}$

$\begin{array}{llll}\mathrm{H} & 17.330699000 & -23.104575000 & 0.256548000\end{array}$

$\begin{array}{llll}\mathrm{H} & 15.946411000 & -23.963842000 & 0.700413000\end{array}$

$\mathrm{H} \quad 21.539331000 \quad-25.715185000 \quad 0.714627000$

$\begin{array}{llll}\mathrm{H} & 23.255556000 & -26.008112000 & 2.484495000\end{array}$

$\mathrm{H} \quad 23.440242000 \quad-28.173939000 \quad 3.692752000$

$\mathrm{H} \quad 21.898256000 \quad-30.038461000 \quad 3.116215000$

H $\quad 20.188749000 \quad-29.733661000 \quad 1.351972000$ 


$\begin{array}{llll}\mathrm{H} & 20.191846000 & -29.382738000 & -2.262645000 \\ \mathrm{H} & 19.884015000 & -28.340722000 & -3.658155000 \\ \mathrm{H} & 21.572273000 & -29.850074000 & -4.129364000 \\ \mathrm{H} & 19.482453000 & -25.710466000 & -2.948735000 \\ \mathrm{Cl} & 18.422375000 & -23.353125000 & -2.396931000\end{array}$

\section{Adduct XXVI}

01

C $\quad 18.061222000 \quad-24.516315000 \quad-2.648438000$

$\begin{array}{llll}\text { C } & 19.507276000 & -24.614261000 & -2.567301000\end{array}$

N $\quad 20.197273000 \quad-25.162100000 \quad-1.630620000$

C $\quad 19.477424000 \quad-25.826727000 \quad-0.542144000$

$\begin{array}{llll}\text { C } & 17.966799000 & -25.471577000 & -0.385493000\end{array}$

$\begin{array}{llll}\text { C } & 17.290992000 & -24.935474000 & -1.613178000\end{array}$

$\mathrm{N} \quad 19.637692000 \quad-27.282625000 \quad-0.587682000$

C $\quad 19.246721000 \quad-27.919904000 \quad-1.850565000$

$\begin{array}{llll}\text { C } & 20.888569000 & -27.777664000 & -0.003708000\end{array}$

N $\quad 15.915183000 \quad-24.965473000 \quad-1.605389000$

C $\quad 18.909859000 \quad-29.393357000 \quad-1.696300000$

C $\quad 18.154898000 \quad-29.855349000 \quad-0.609918000$

C $\quad 17.801236000 \quad-31.200098000 \quad-0.509613000$

C $\quad 18.194102000 \quad-32.106405000 \quad-1.497357000$

$\begin{array}{llll}\text { C } & 18.948331000 & -31.657802000 & -2.581385000\end{array}$

$\begin{array}{llll}\text { C } & 19.305851000 & -30.311416000 & -2.675114000\end{array}$

C $\quad 22.195147000 \quad-27.415593000 \quad-0.721999000$

O $\quad 23.229491000 \quad-28.093695000 \quad-0.010239000$

$\mathrm{H} \quad 17.614136000 \quad-24.099783000 \quad-3.546274000$

$\mathrm{H} \quad 20.072708000 \quad-24.174563000 \quad-3.393302000$

$\mathrm{H} \quad 17.446629000 \quad-26.351995000 \quad-0.006361000$

$\mathrm{H} \quad 18.354435000 \quad-27.397659000 \quad-2.216348000$

$\mathrm{H} \quad 20.001702000 \quad-27.803709000 \quad-2.642858000$

$\mathrm{H} \quad 20.953112000 \quad-27.415201000 \quad 1.028676000$

H $\quad 20.825698000 \quad-28.868748000 \quad 0.050396000$

$\begin{array}{llll}\mathrm{H} & 15.476851000 & -24.874474000 & -0.698146000\end{array}$

$\mathrm{H} \quad 15.451037000 \quad-24.431377000 \quad-2.327418000$

$\mathrm{H} \quad 17.863595000 \quad-29.151759000 \quad 0.164136000$

$\begin{array}{llll}\mathrm{H} & 17.218831000 & -31.542535000 & 0.341285000\end{array}$

$\mathrm{H} \quad 17.919158000 \quad-33.154222000 \quad-1.418479000$

$\mathrm{H} \quad 19.266446000 \quad-32.355664000 \quad-3.350763000$

$\mathrm{H} \quad 19.900432000 \quad-29.968657000 \quad-3.518657000$

$\mathrm{H} \quad 22.155587000 \quad-27.740691000 \quad-1.773153000$

$\mathrm{H} \quad 22.341179000 \quad-26.328451000 \quad-0.724793000$

$\mathrm{H} \quad 24.074824000 \quad-27.822605000 \quad-0.388256000$

$\begin{array}{llll}\mathrm{H} & 19.961974000 & -25.509481000 & 0.385021000\end{array}$

$\begin{array}{llll}\mathrm{Cl} & 17.732583000 & -24.214623000 & 0.965656000\end{array}$

\section{Adduct XXVII}

01

C $\quad 18.732572000 \quad-23.953744000 \quad-2.052100000$

C $\quad 20.005507000 \quad-24.201301000 \quad-1.399713000$

N $\quad 20.269984000 \quad-25.114822000 \quad-0.534183000$ 


$\begin{array}{llll}\mathrm{C} & 19.212376000 & -26.068864000 & -0.193484000 \\ \mathrm{C} & 17.756868000 & -25.664164000 & -0.580900000 \\ \mathrm{C} & 17.631321000 & -24.656101000 & -1.685154000 \\ \mathrm{~N} & 19.500900000 & -27.412338000 & -0.702916000 \\ \mathrm{C} & 19.746468000 & -27.498515000 & -2.147318000 \\ \mathrm{C} & 20.417375000 & -28.197370000 & 0.130550000 \\ \mathrm{~N} & 16.394212000 & -24.566092000 & -2.281185000 \\ \mathrm{C} & 19.507776000 & -28.888863000 & -2.711363000 \\ \mathrm{C} & 18.403406000 & -29.653824000 & -2.312747000 \\ \mathrm{C} & 18.161220000 & -30.903765000 & -2.880583000 \\ \mathrm{C} & 19.018556000 & -31.409156000 & -3.860850000 \\ \mathrm{C} & 20.122341000 & -30.657351000 & -4.262914000 \\ \mathrm{C} & 20.365074000 & -29.408674000 & -3.687342000 \\ \mathrm{C} & 21.869670000 & -27.713078000 & 0.232529000 \\ \mathrm{O} & 22.551952000 & -28.695178000 & 1.011163000 \\ \mathrm{H} & 18.681112000 & -23.197670000 & -2.830042000 \\ \mathrm{H} & 20.830881000 & -23.538950000 & -1.674263000 \\ \mathrm{H} & 17.201134000 & -26.572612000 & -0.816135000 \\ \mathrm{H} & 19.054302000 & -26.803223000 & -2.637401000 \\ \mathrm{H} & 20.754381000 & -27.161375000 & -2.433102000 \\ \mathrm{H} & 20.001573000 & -28.249720000 & 1.143328000 \\ \mathrm{H} & 20.430584000 & -29.220811000 & -0.256357000 \\ \mathrm{H} & 15.602218000 & -24.778677000 & -1.688689000 \\ \mathrm{H} & 16.241376000 & -23.764649000 & -2.878131000 \\ \mathrm{H} & 17.746898000 & -29.265303000 & -1.540014000 \\ \mathrm{H} & 17.302548000 & -31.485727000 & -2.557335000 \\ \mathrm{H} & 18.830084000 & -32.383412000 & -4.302483000 \\ \mathrm{H} & 20.800257000 & -31.044560000 & -5.018328000 \\ \mathrm{H} & 21.231454000 & -28.830180000 & -3.999268000 \\ \mathrm{H} & 22.312882000 & -27.621990000 & -0.771180000 \\ \mathrm{H} & 21.909630000 & -26.719430000 & 0.695416000 \\ \mathrm{H} & 23.451257000 & -28.379859000 & 1.162151000 \\ \mathrm{H} & 19.222235000 & -26.162271000 & 0.895658000 \\ \mathrm{Cl} & 16.859433000 & -24.977213000 & 0.896570000\end{array}$

\section{Adduct XXVIII}

$\begin{array}{llll}\text { 0 1 } & & & \\ \mathrm{C} & 16.702215000 & -24.622095000 & -1.350059000 \\ \mathrm{C} & 16.546048000 & -25.936735000 & -1.767167000 \\ \mathrm{~N} & 17.483199000 & -26.883305000 & -1.676116000 \\ \mathrm{C} & 18.678753000 & -26.548080000 & -1.147461000 \\ \mathrm{C} & 18.948630000 & -25.229297000 & -0.706974000 \\ \mathrm{C} & 17.946393000 & -24.270254000 & -0.806097000 \\ \mathrm{~N} & 19.623974000 & -27.548454000 & -1.062007000 \\ \mathrm{C} & 19.360071000 & -28.889244000 & -1.599934000 \\ \mathrm{C} & 20.903725000 & -27.348443000 & -0.400290000 \\ \mathrm{~N} & 18.161584000 & -22.961407000 & -0.313201000 \\ \mathrm{C} & 19.070006000 & -29.931014000 & -0.533643000 \\ \mathrm{C} & 17.929445000 & -29.820540000 & 0.275854000 \\ \mathrm{C} & 17.659977000 & -30.777725000 & 1.251603000\end{array}$




$\begin{array}{lrrr}\mathrm{C} & 18.524620000 & -31.861060000 & 1.434336000 \\ \mathrm{C} & 19.659178000 & -31.981137000 & 0.633675000 \\ \mathrm{C} & 19.928342000 & -31.019007000 & -0.343300000 \\ \mathrm{C} & 21.985229000 & -26.801171000 & -1.336653000 \\ \mathrm{O} & 23.167577000 & -26.662328000 & -0.561559000 \\ \mathrm{H} & 15.893661000 & -23.904058000 & -1.435234000 \\ \mathrm{H} & 15.599569000 & -26.254897000 & -2.201382000 \\ \mathrm{H} & 19.913945000 & -24.953308000 & -0.300458000 \\ \mathrm{H} & 18.513159000 & -28.798342000 & -2.280236000 \\ \mathrm{H} & 20.233183000 & -29.202923000 & -2.185271000 \\ \mathrm{H} & 20.795552000 & -26.680694000 & 0.459720000 \\ \mathrm{H} & 21.238383000 & -28.309044000 & 0.001916000 \\ \mathrm{H} & 19.140598000 & -22.693379000 & -0.300258000 \\ \mathrm{H} & 17.619106000 & -22.258396000 & -0.804912000 \\ \mathrm{H} & 17.254296000 & -28.983181000 & 0.126854000 \\ \mathrm{H} & 16.772026000 & -30.681828000 & 1.870079000 \\ \mathrm{H} & 18.312528000 & -32.605711000 & 2.196081000 \\ \mathrm{H} & 20.337058000 & -32.819179000 & 0.768636000 \\ \mathrm{H} & 20.813348000 & -31.118140000 & -0.968028000 \\ \mathrm{H} & 22.125974000 & -27.497736000 & -2.178036000 \\ \mathrm{H} & 21.659228000 & -25.839383000 & -1.762297000 \\ \mathrm{H} & 23.878596000 & -26.364176000 & -1.141908000 \\ \mathrm{H} & 17.644471000 & -22.747342000 & 1.395411000 \\ \mathrm{Cl} & 17.287404000 & -22.483548000 & 2.653728000\end{array}$

\section{Adduct XXIX}

$\begin{array}{lccc}\text { O } 1 & & & \\ \mathrm{C} & 16.649875000 & -24.651069000 & -0.605182000 \\ \mathrm{C} & 16.718459000 & -25.869450000 & 0.056257000 \\ \mathrm{~N} & 17.724464000 & -26.740746000 & -0.050304000 \\ \mathrm{C} & 18.754585000 & -26.428741000 & -0.864378000 \\ \mathrm{C} & 18.775510000 & -25.218253000 & -1.599424000 \\ \mathrm{C} & 17.716695000 & -24.327968000 & -1.456757000 \\ \mathrm{~N} & 19.780666000 & -27.346036000 & -0.949284000 \\ \mathrm{C} & 19.706797000 & -28.633910000 & -0.247279000 \\ \mathrm{C} & 21.001673000 & -27.073712000 & -1.691341000 \\ \mathrm{~N} & 17.739716000 & -23.080746000 & -2.124777000 \\ \mathrm{C} & 20.579045000 & -28.706047000 & 0.993870000 \\ \mathrm{C} & 20.318598000 & -27.875794000 & 2.094426000 \\ \mathrm{C} & 21.115723000 & -27.943460000 & 3.235004000 \\ \mathrm{C} & 22.185362000 & -28.841707000 & 3.295345000 \\ \mathrm{C} & 22.450993000 & -29.672487000 & 2.208173000 \\ \mathrm{C} & 21.651097000 & -29.601827000 & 1.064587000 \\ \mathrm{C} & 20.909142000 & -27.472454000 & -3.167256000 \\ \mathrm{O} & 22.161768000 & -27.155595000 & -3.757600000 \\ \mathrm{H} & 15.811279000 & -23.978249000 & -0.461434000 \\ \mathrm{H} & 15.910569000 & -26.166080000 & 0.723278000 \\ \mathrm{H} & 19.591904000 & -24.974888000 & -2.268411000 \\ \mathrm{H} & 18.661002000 & -28.793889000 & 0.015465000 \\ \mathrm{H} & 20.005540000 & -29.424302000 & -0.946823000\end{array}$




$\begin{array}{llll}\mathrm{H} & 21.273374000 & -26.016453000 & -1.616600000 \\ \mathrm{H} & 21.820109000 & -27.630671000 & -1.226056000 \\ \mathrm{H} & 18.305555000 & -23.087300000 & -2.967546000 \\ \mathrm{H} & 16.812096000 & -22.727038000 & -2.336186000 \\ \mathrm{H} & 19.482168000 & -27.184767000 & 2.049197000 \\ \mathrm{H} & 20.901746000 & -27.296827000 & 4.081318000 \\ \mathrm{H} & 22.805620000 & -28.892198000 & 4.185556000 \\ \mathrm{H} & 23.280164000 & -30.373284000 & 2.245724000 \\ \mathrm{H} & 21.859981000 & -30.254219000 & 0.219548000 \\ \mathrm{H} & 20.681278000 & -28.547421000 & -3.241064000 \\ \mathrm{H} & 20.080414000 & -26.930941000 & -3.649402000 \\ \mathrm{H} & 22.145502000 & -27.443153000 & -4.678629000 \\ \mathrm{H} & 18.462082000 & -21.758601000 & -1.143714000 \\ \mathrm{Cl} & 18.973819000 & -20.709880000 & -0.496603000\end{array}$

\section{Adduct XXX}

$\begin{array}{lrrr}\text { O } 1 & & & \\ \mathrm{C} & 17.033884000 & -24.396810000 & -1.759173000 \\ \mathrm{C} & 17.418048000 & -25.267699000 & -2.769433000 \\ \mathrm{~N} & 18.294730000 & -26.264407000 & -2.626171000 \\ \mathrm{C} & 18.862540000 & -26.445007000 & -1.415307000 \\ \mathrm{C} & 18.553741000 & -25.600821000 & -0.320757000 \\ \mathrm{C} & 17.627884000 & -24.578967000 & -0.501535000 \\ \mathrm{~N} & 19.759514000 & -27.485706000 & -1.297510000 \\ \mathrm{C} & 20.132872000 & -28.300549000 & -2.460874000 \\ \mathrm{C} & 20.342755000 & -27.862780000 & -0.019515000 \\ \mathrm{~N} & 17.247300000 & -23.761386000 & 0.588917000 \\ \mathrm{C} & 19.492569000 & -29.677598000 & -2.475197000 \\ \mathrm{C} & 18.100300000 & -29.812621000 & -2.583521000 \\ \mathrm{C} & 17.511960000 & -31.075343000 & -2.600641000 \\ \mathrm{C} & 18.304349000 & -32.223655000 & -2.510647000 \\ \mathrm{C} & 19.688666000 & -32.100286000 & -2.405100000 \\ \mathrm{C} & 20.276492000 & -30.832719000 & -2.386474000 \\ \mathrm{C} & 21.630489000 & -27.098406000 & 0.301981000 \\ \mathrm{O} & 22.083183000 & -27.568848000 & 1.563616000 \\ \mathrm{H} & 16.301159000 & -23.616785000 & -1.935993000 \\ \mathrm{H} & 16.988255000 & -25.161298000 & -3.764195000 \\ \mathrm{H} & 19.022898000 & -25.731644000 & 0.646736000 \\ \mathrm{H} & 19.842704000 & -27.739169000 & -3.349130000 \\ \mathrm{H} & 21.224521000 & -28.408150000 & -2.469238000 \\ \mathrm{H} & 19.623201000 & -27.720463000 & 0.792382000 \\ \mathrm{H} & 20.564665000 & -28.933624000 & -0.043736000 \\ \mathrm{H} & 17.957770000 & -23.699596000 & 1.311339000 \\ \mathrm{H} & 16.963337000 & -22.829069000 & 0.305306000 \\ \mathrm{H} & 17.486948000 & -28.919977000 & -2.662056000 \\ \mathrm{H} & 16.432891000 & -31.166101000 & -2.687469000 \\ \mathrm{H} & 17.843160000 & -33.206954000 & -2.523730000 \\ \mathrm{H} & 20.312469000 & -32.986790000 & -2.334177000 \\ \mathrm{H} & 21.357510000 & -30.741024000 & -2.306899000 \\ \mathrm{H} & 22.370719000 & -27.280011000 & -0.493009000\end{array}$




$\begin{array}{llll}\mathrm{H} & 21.428702000 & -26.016019000 & 0.316778000 \\ \mathrm{H} & 22.921788000 & -27.136613000 & 1.766501000 \\ \mathrm{H} & 15.803872000 & -24.372218000 & 1.469614000 \\ \mathrm{Cl} & 14.737903000 & -24.736458000 & 2.184804000\end{array}$

\section{Adduct XXXI}

$\begin{array}{lrrr}0 & 1 & & \\ \mathrm{C} & 17.033884000 & -24.396810000 & -1.759173000 \\ \mathrm{C} & 17.418048000 & -25.267699000 & -2.769433000 \\ \mathrm{~N} & 18.294730000 & -26.264407000 & -2.626171000 \\ \mathrm{C} & 18.862540000 & -26.445007000 & -1.415307000 \\ \mathrm{C} & 18.553741000 & -25.600821000 & -0.320757000 \\ \mathrm{C} & 17.627884000 & -24.578967000 & -0.501535000 \\ \mathrm{~N} & 19.759514000 & -27.485706000 & -1.297510000 \\ \mathrm{C} & 20.132872000 & -28.300549000 & -2.460874000 \\ \mathrm{C} & 20.342755000 & -27.862780000 & -0.019515000 \\ \mathrm{~N} & 17.247300000 & -23.761386000 & 0.588917000 \\ \mathrm{C} & 19.492569000 & -29.677598000 & -2.475197000 \\ \mathrm{C} & 18.100300000 & -29.812621000 & -2.583521000 \\ \mathrm{C} & 17.511960000 & -31.075343000 & -2.600641000 \\ \mathrm{C} & 18.304349000 & -32.223655000 & -2.510647000 \\ \mathrm{C} & 19.688666000 & -32.100286000 & -2.405100000 \\ \mathrm{C} & 20.276492000 & -30.832719000 & -2.386474000 \\ \mathrm{C} & 21.630489000 & -27.098406000 & 0.301981000 \\ \mathrm{O} & 22.083183000 & -27.568848000 & 1.563616000 \\ \mathrm{H} & 16.301159000 & -23.616785000 & -1.935993000 \\ \mathrm{H} & 16.988255000 & -25.161298000 & -3.764195000 \\ \mathrm{H} & 19.022898000 & -25.731644000 & 0.646736000 \\ \mathrm{H} & 19.842704000 & -27.739169000 & -3.349130000 \\ \mathrm{H} & 21.224521000 & -28.408150000 & -2.469238000 \\ \mathrm{H} & 19.623201000 & -27.720463000 & 0.792382000 \\ \mathrm{H} & 20.564665000 & -28.933624000 & -0.043736000 \\ \mathrm{H} & 17.957770000 & -23.699596000 & 1.311339000 \\ \mathrm{H} & 16.963337000 & -22.829069000 & 0.305306000 \\ \mathrm{H} & 17.486948000 & -28.919977000 & -2.662056000 \\ \mathrm{H} & 16.432891000 & -31.166101000 & -2.687469000 \\ \mathrm{H} & 17.843160000 & -33.206954000 & -2.523730000 \\ \mathrm{H} & 20.312469000 & -32.986790000 & -2.334177000 \\ \mathrm{H} & 21.357510000 & -30.741024000 & -2.306899000 \\ \mathrm{H} & 22.370719000 & -27.280011000 & -0.493009000 \\ \mathrm{H} & 21.428702000 & -26.016019000 & 0.316778000 \\ \mathrm{H} & 22.921788000 & -27.136613000 & 1.766501000 \\ \mathrm{H} & 15.803872000 & -24.372218000 & 1.469614000 \\ \mathrm{Cl} & 14.737903000 & -24.736458000 & 2.184804000\end{array}$


